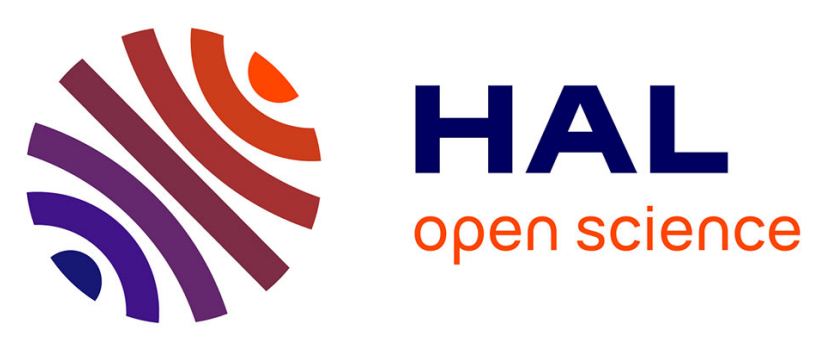

\title{
Double scale analysis of a Schrödinger-Poisson system with quantum wells and macroscopic nonlinearities in dimensions 2 and 3
}

\author{
Ali Faraj, Andrea Mantile, Francis Nier
}

\section{- To cite this version:}

Ali Faraj, Andrea Mantile, Francis Nier. Double scale analysis of a Schrödinger-Poisson system with quantum wells and macroscopic nonlinearities in dimensions 2 and 3. 2008. hal-00276246

\section{HAL Id: hal-00276246 \\ https://hal.science/hal-00276246}

Preprint submitted on 29 Apr 2008

HAL is a multi-disciplinary open access archive for the deposit and dissemination of scientific research documents, whether they are published or not. The documents may come from teaching and research institutions in France or abroad, or from public or private research centers.
L'archive ouverte pluridisciplinaire HAL, est destinée au dépôt et à la diffusion de documents scientifiques de niveau recherche, publiés ou non, émanant des établissements d'enseignement et de recherche français ou étrangers, des laboratoires publics ou privés. 


\title{
Double scale analysis of a Schrödinger-Poisson system with quantum wells and macroscopic nonlinearities in dimensions 2 and 3.
}

\author{
A. Faraj, A. Mantileł F. Nier ${ }^{\ddagger}$
}

\begin{abstract}
We consider the stationary Schrödinger-Poisson model with a background potential describing a quantum well. The Hamiltonian of this system composes of contributions - the background potential well plus a nonlinear repulsive term - which extends on different length scales with ratio parametrized by the small parameter $h$. With a partition function which forces the particles to remain in the quantum well, the limit $h \rightarrow 0$ in the nonlinear system leads to different asymptotic behaviours, including spectral renormalization, depending on the dimensions 1,2 or 3 .
\end{abstract}

\section{Introduction}

The quantum state of a gas of charged particles is described, in the mean field approximation, by a nonlinear one-particle Schrödinger equation where the electrostatic repulsion is modeled by a non linear potential term depending on the charge density through a Poisson equation. This class of models is usually referred to as Schrödinger-Poisson systems. In this work we consider a stationary Schrödinger-Poisson system in a bounded region of $\mathbb{R}^{d}, d=2,3$, for which a background potential models a quantum well, while the nonlinear potential extends on a wider scale. After introducing a rescaling for which the small parameter $h>0$ represents an inverse length scale, the support of the potential well squeezes asymptotically to a single point in the limit $h \rightarrow 0$. An equilibrium state of a gas of charged particles confined in the quantum well will be considered, while the nonlinear electrostatic potential created by such a concentrated charge extends to whole domain with different behaviour far from the well according to the dimension 1, 2 or 3 .

Such a Schrödinger-Poisson problem has recently been considered in [2], [3] and [12] in a more complex - although 1 dimensional - setting involving far from equilibrium steady states. This onedimensional analysis leads to a reduced model which happens to be very efficient in the numerical simulation of the electronic transport through semiconductor heterostructures, like resonant tunneling diodes [4]. In particular this technique allows to forecast with high precision the nonlinear phenomenology - like hysteresis phenomena (e.g. in [10] and [16]) and steady oscillating currents (e.g. in [11]) - observed in such devices. A first step in the extension of this analysis to the multidimensional case consists in a good understanding of the thermodynamical equilibrium where the occupation numbers of the quantum states are given by a decreasing function of the energy.

For the sake of simplicity we shall use a low energy-filter in the definition of the partition function $f$ (see equation (1.5) below), that is the quantum states with an energy larger than the threshold $\varepsilon_{S}$ are not occupied. With such an assumption only the quantum states confined in the well have an effect on the nonlinearity. This provides asymptotically a strict separation of the quantum and macroscopic scales with some nonlinear spectral renormalization which depends on the dimension $d=2$ or $d=3$.

\footnotetext{
*IMT, UMR - CNRS 5219, Université Paul Sabatier, 31062 Toulouse Cedex 9, France

${ }^{\dagger}$ IRMAR, UMR - CNRS 6625, Université Rennes 1, Campus de Beaulieu, 35042 Rennes Cedex, France.

${ }^{\ddagger}$ IRMAR, UMR - CNRS 6625, Université Rennes 1, Campus de Beaulieu, 35042 Rennes Cedex, France.
} 
Like in [2], [3] and [13] the analysis will be a mixture of nonlinear apriori estimates combined with accurate semiclassical and spectral techniques (we refer to: [17], [8], [9] and [6]) adapted for potentials with limited regularity. The outline of this analysis is the following. We end this section by introducing the model - both at the macroscopic and quantum scales - and by stating our results. In Section 2, apriori estimates for the nonlinear problem are given. The Section 3 studies the possible asymptotic nonlinear system in dimension $d=3$ and ends the proof of the main result in this case. The analysis of the bidimensional problem is completed in Section 4, with a different renormalization process. Some standard results are adapted to our case in the appendices.

\subsection{The model}

Let $\Omega$ be an open bounded set of $\mathbb{R}^{d}, d \leq 3$, and $U$ a non positive function in $C_{0}^{\infty}\left(\mathbb{R}^{d}\right)$ supported in the ball of radius one centered in the origin of $\mathbb{R}^{d}$. The open set $\Omega$ is supposed smooth enough (for example $C^{2}$ or piecewise $C^{1}$ and convex) so that the domain of the Dirichlet Laplacian is $H^{2} \cap H_{0}^{1}(\Omega)$.

For $x_{0} \in \Omega$, we define the potential with center $x_{0}$ and radius of order $h>0$

$$
U^{h}(x)=U\left(\frac{x-x_{0}}{h}\right), \quad x \in \Omega .
$$

Being our analysis concerned with the limit $h \rightarrow 0$, we can choose, without loss of generality, $h$ small enough so that the support of $U^{h}$ is included in $\Omega$. In particular, defining with $\omega$ and $\omega^{h}$ the supports of $U$ and $U^{h}$ respectively, we assume that $\omega^{h} \subset \Omega$ for all values of $h$ below a suitable positive constant: $h \leq h_{0}$.

Next we assign the function $f \in C^{\infty}(\mathbb{R})$, with a threshold at $\varepsilon_{S}<0$ and fulfilling the conditions

$$
\begin{aligned}
& f(x)>0, \quad \forall x<\varepsilon_{S}, \\
& f(x)=0, \quad \forall x \geq \varepsilon_{S}, \\
& f^{\prime}(x) \leq 0, \quad \forall x \in \mathbb{R},
\end{aligned}
$$

and address, for $h \in\left(0, h_{0}\right]$, the following problem: find $V^{h}$ solving the non-linear Poisson equation

$$
\left\{\begin{array}{l}
-\Delta V^{h}=n\left[V^{h}\right] \quad \text { in } \Omega \\
\left.V^{h}\right|_{\partial \Omega}=0
\end{array}\right.
$$

where the source term is

$$
n\left[V^{h}\right]=\sum_{i \in \mathbb{N}} f\left(\varepsilon_{i}^{h}\right)\left|\Psi_{i}^{h}\right|^{2},
$$

with $\left\{\varepsilon_{i}^{h}\right\}_{i \in \mathbb{N}}$ given by the eigenvalues of the nonlinear Hamiltonian

$$
H^{h}=-h^{2} \Delta+U^{h}+V^{h}
$$

numerated from $\inf \sigma\left(H^{h}\right)$ counting multiplicities, while $\left\{\Psi_{i}^{h}\right\}_{i \in \mathbb{N}}$ are the corresponding eigenvectors

$$
\left\{\begin{array}{l}
H^{h} \Psi_{i}^{h}=\varepsilon_{i}^{h} \Psi_{i}^{h}, \quad \text { in } \Omega \\
\left.\Psi_{i}^{h}\right|_{\partial \Omega}=0
\end{array}\right.
$$

The equations (1.4), (1.5) and (1.7) define the stationary Schrödinger-Poisson system associated with the potential well $U^{h}$ and the function $f$. In practical applications, where these equations are used for the description of the charge distribution in electronic devices, $n\left[V^{h}\right]$ describes the density of the charge careers of the system, while $f$ is a response function which depends on the characteristics of the device and has to be considered as a data item of the problem.

The small parameter $h>0$ arises from a rescaling after considering two length scales, the macroscopic one where the particles behave like classical particles and the microscopic one where the quantum effects have to be taken into account. From this point of view it should be noticed 
that on $\mathbb{R}^{d}$, the Schrödinger operator $-h^{2} \Delta+U\left(\frac{x-x_{0}}{h}\right)$ is unitarily equivalent to $-\Delta+U(x)$ through the unitary map: $L^{2}(\Omega) \rightarrow L^{2}\left(\Omega^{h}\right)$

$$
\tilde{\Psi}^{h}(x)=h^{\frac{d}{2}} \Psi^{h}\left(h x+x_{0}\right), \quad x \in \Omega^{h},
$$

with

$$
\Omega^{h}=\left\{x \in \mathbb{R}^{d} \mid h x+x_{0} \in \Omega\right\} .
$$

It is easy to show that, under this transformation, the system of equations (1.4) - (1.7) reads as

$$
\left\{\begin{array}{l}
\left(-\Delta+U+\tilde{V}^{h}\right) \tilde{\Psi}_{i}^{h}=\varepsilon_{i}^{h} \tilde{\Psi}_{i}^{h} \quad \text { in } \Omega^{h} \\
-\Delta \tilde{V}^{h}=h^{2-d} \sum_{i \in \mathbb{N}} f\left(\varepsilon_{i}^{h}\right)\left|\tilde{\Psi}_{i}^{h}\right|^{2} \quad \text { in } \Omega^{h} \\
\left.\tilde{V}^{h}\right|_{\partial \Omega^{h}}=0,\left.\quad \tilde{\Psi}_{i}^{h}\right|_{\partial \Omega^{h}}=0
\end{array}\right.
$$

where the Poisson term is related to $V^{h}$ by

$$
\tilde{V}^{h}(x)=V^{h}\left(h x+x_{0}\right), \quad x \in \Omega^{h} .
$$

In this picture, the parameter $h$ defines an isotropic dilation of the domain $\Omega^{h}$ such that, in the limit $h \rightarrow 0, \Omega^{h}$ covers the whole space $\mathbb{R}^{d}$. We will refer to (1.10) as the Schrödinger-Poisson problem at the quantum scale, while the equations (1.4) - (1.7) describe the problem at the classical scale. In both settings, the stationary states form a set of real normalized functions

$$
\begin{aligned}
& \operatorname{Im} \Psi_{i}^{h}=0 ;\left\|\Psi_{i}^{h}\right\|_{L^{2}(\Omega)}=1, \\
& \operatorname{Im} \tilde{\Psi}_{i}^{h}=0 ;\left\|\tilde{\Psi}_{i}^{h}\right\|_{L^{2}\left(\Omega^{h}\right)}=1 .
\end{aligned}
$$

The analysis of our Schrödinger-Poisson system, will involve the operator

$$
H_{0}=-\Delta+U ; \quad D\left(H_{0}\right)=H^{2}\left(\mathbb{R}^{d}\right),
$$

whose point spectrum, $\sigma_{p}\left(H_{0}\right)$, contains a finite number of points embedded in $\left[-\|U\|_{L^{\infty}}, 0\right)$. In particular, we make the following assumptions

$$
\sigma_{p}\left(H_{0}\right) \neq \varnothing
$$

and

$$
e_{1}:=\inf \sigma\left(H_{0}\right)<\varepsilon_{S} .
$$

The reader may refer to Proposition 7.4 in [18] to see that (1.15) is always true for $d=1,2$ and $U<0$. On the other hand, for $d=3$, potentials fulfilling this condition can be obtained by possibly replacing $U \leq 0$ with $\lambda U \leq 0, \lambda>1$ large enough. The hypothesis (1.16) - which prevents the solution to (1.4) - (1.7) to be trivial - will be extensively used in this work.

\subsection{Results}

The aim of this analysis is to understand the asymptotic behaviour of the unitarily equivalent systems (1.4) - (1.7) and (1.10) as $h \rightarrow 0$. This in order to provide a simplified modelling for the nonlinearities produced by charged particles confined in quantum wells $(d=3)$, wires $(d=2)$ or layers $(d=1)$. Such a program has been carried out in [2], [3] and [13] with efficient numerical applications in [4] for out-of equilibrium $1 D$ problem. A variation of it (actually simpler than the analysis in [2][3][13] for no scattering nor resonant states have to be considered here) provides the result for the present $1 D$-Dirichlet problem with $\Omega=(0, L)$ : the Poisson potential $V^{h}$ is uniformly bounded in $W^{1, \infty}(\Omega)$ and converges in $C^{0, \alpha}(\Omega), \alpha \in(0,1)$, to $V_{0}$ defined by

$$
V_{0}(x)= \begin{cases}\left(\sum_{i \geq 1} f\left(e_{i}+\beta\right)\right)\left(1-\frac{x_{0}}{L}\right) x, & 0<x \leq x_{0}, \\ \left(\sum_{i \geq 1} f\left(e_{i}+\beta\right)\right) \frac{x_{0}}{L}(L-x), & x_{0}<x<L,\end{cases}
$$


where $\left\{e_{i}, i \geq 1\right\}$ is the point spectrum of $H_{0}$ and $\beta$ is the unique solution to the nonlinear equation $\beta=x_{0}\left(1-\frac{x_{0}}{L}\right) \sum_{i \geq 1} f\left(e_{i}+\beta\right)$.

Hence for the $1 D$ problem, the nonlinear effect produced at the quantum scale remain visible at the macroscopic scale in the limit $h \rightarrow 0$. This is no more the case in dimension $d>1$. Indeed, due to the different behaviour of the Green function of the Laplace operator in dimension $d=2$ and $d=3$, the potential at the classical scale $V^{h}$ is expected to converge to 0 as $h \rightarrow 0$, although a simple ad absurdum argument shows that some nonlinearity still affects asymptotically the spectrum of the quantum Hamiltonian. The complete description of this requires the analysis of the asymptotic behaviour of both $V^{h}$ and $\tilde{V}^{h}$. Again the differences of the Green functions of the Laplace operator in dimension $d=2$ and $d=3$ require different kind of arguments and lead to different results: a renormalization of the logarithmic divergence has to be introduced in dimension 2 , not in dimension 3.

Our main results, whose proofs are given in Sections 3 and 4, gather the asymptotic information for the $3 D$ and $2 D$ cases.

Theorem 1.1 Let $d=3$ and let $V^{h}$ (resp. $\left.\tilde{V}^{h}\right)$ solve (1.4)(1.5)(1.7) (resp. (1.10))

1. The potential at the classical scale, $V^{h}$, converges strongly to 0 in $H_{0}^{1}(\Omega)$ :

$$
\left\|V^{h}\right\|_{H_{0}^{1}(\Omega)}=\mathcal{O}\left(h^{1 / 2}\right) .
$$

2. By fixing the threshold $\varepsilon_{S}$ associated with $f$, there exists a unique $(A, W) \in(0,+\infty) \times$ $\dot{H}^{1}\left(\mathbb{R}^{3} ; \mathbb{R}\right)$ such that $\varepsilon_{S}=\inf \sigma(-\Delta+U+W)$ and

$$
\left\{\begin{array}{l}
{[-\Delta+U+W] \chi=\varepsilon_{S} \chi, \text { with } \quad \chi \in H^{2}\left(\mathbb{R}^{3}\right),\|\chi\|_{L^{2}\left(\mathbb{R}^{3}\right)}=1,} \\
-\Delta W=A|\chi|^{2} .
\end{array}\right.
$$

3. With above notations, the potential at the quantum scale $\tilde{V}^{h}$ satisfies

$$
\lim _{h \rightarrow 0}\left\|1_{\Omega^{h}} \tilde{V}^{h}-W\right\|_{L^{\infty}\left(\mathbb{R}^{3}\right)}=0 .
$$

4. There exists $h_{1}>0$ such that the eigenvalues $\varepsilon_{i}^{h}$ are larger than $\varepsilon_{S}$ and $f\left(\varepsilon_{i}^{h}\right)=0$ for all $i \geq 2$ and all $h \leq h_{1}$. The particle density at the quantum scale, $h^{-1} \sum_{i \in \mathbb{N}} f\left(\varepsilon_{i}^{h}\right)\left|\tilde{\Psi}_{i}^{h}\right|^{2}=$ $h^{-1} f\left(\varepsilon_{1}^{h}\right)\left|\tilde{\Psi}_{1}^{h}\right|^{2}$ for $h \leq h_{1}$, satisfies

$$
\lim _{h \rightarrow 0}\left\|1_{\Omega^{h}} h^{-1} f\left(\varepsilon_{1}^{h}\right)\left|\tilde{\Psi}_{1}^{h}\right|^{2}-A|\chi|^{2}\right\|_{L^{1} \cap L^{2}\left(\mathbb{R}^{3}\right)}=0 .
$$

Remark 1.2 The third statement prevents $V^{h}(x)=\tilde{V}^{h}\left(\frac{x-x_{0}}{h}\right)$ from converging to 0 in the $L^{\infty}$ norm.

Theorem 1.3 Let $d=2$ and let $V^{h}$ (resp. $\left.\tilde{V}^{h}\right)$ solve (1.4)(1.5)(1.7) (resp. (1.10))

1. The potential at the classical scale, $V^{h}$, converges strongly to 0 in $H_{0}^{1}(\Omega)$

$$
\left\|V^{h}\right\|_{H_{0}^{1}(\Omega)}=\mathcal{O}\left(\frac{1}{|\ln h|}\right) .
$$

2. Take the threshold $\varepsilon_{S}$ associated with $f$ and $e_{1}=\inf \sigma(-\Delta+U)$ and $\operatorname{set} \theta=\varepsilon_{S}-e_{1}$. Then the potential $\tilde{V}^{h}$ at the quantum scale satisfies

$$
\lim _{h \rightarrow 0}\left\|\tilde{V}^{h}-\theta\right\|_{L^{\infty}(\{|x| \leq-\kappa \ln h\})}=0
$$

for any fixed $\kappa>0$. 
3. There exists $h_{1}>0$ such that the eigenvalues $\varepsilon_{i}^{h}$ are larger than $\varepsilon_{S}$ and $f\left(\varepsilon_{i}^{h}\right)=0$ for all $i \geq 2$ and all $h \leq h_{1}$. The particle density at the quantum scale, $\sum_{i \in \mathbb{N}} f\left(\varepsilon_{i}^{h}\right)\left|\tilde{\Psi}_{i}^{h}\right|^{2}=f\left(\varepsilon_{1}^{h}\right)\left|\tilde{\Psi}_{1}^{h}\right|^{2}$ for $h \leq h_{1}$, satisfies

$$
\begin{aligned}
& \left\|1_{\Omega^{h}} f\left(\varepsilon_{1}^{h}\right)\left|\tilde{\Psi}_{1}^{h}\right|^{2}\right\|_{L^{2}\left(\mathbb{R}^{2}\right)}=\mathcal{O}\left(|\ln h|^{-1}\right), \\
& \lim _{h \rightarrow 0}|\ln h|\left\|1_{\Omega^{h}} f\left(\varepsilon_{1}^{h}\right)\left|\tilde{\Psi}_{1}^{h}\right|^{2}\right\|_{L^{1}\left(\mathbb{R}^{2}\right)}=\lim _{h \rightarrow 0}|\ln h| f\left(\varepsilon_{1}^{h}\right)=2 \pi \theta .
\end{aligned}
$$

Remark 1.4 Contrarily to the $3 D$ case, the total charge in the quantum well converges to 0 but still has a spectral effect due to the logarithmic divergence of the Green function of the Laplace operator.

\section{Asymptotic estimates in dimension $d=2,3$}

In this Section our investigation is confined to the $2 \mathrm{D}$ and the $3 \mathrm{D}$ case. We give some preliminary results related to the asymptotic behaviour for $h \rightarrow 0$ of the charge density and the eigenvalues related to the Schrödinger-Poisson problem. The classical or the quantum scale pictures will be alternatively adopted depending on the strategies of the proofs. From the lower bounds

$$
-\Delta V^{h} \geq 0 \text { in } \Omega, \text { and } \quad-\Delta \tilde{V}^{h} \geq 0 \text { in } \Omega^{h},
$$

with homogeneous Dirichlet boundary condition, the maximum principle implies

$$
V^{h} \geq 0 \text { in } \Omega \text { and } \tilde{V}^{h} \geq 0 \text { in } \Omega^{h} .
$$

Thus, $V^{h}$ and $\tilde{V}^{h}$ define positive perturbations of the unitarily equivalent Hamiltonians

$$
H_{0}^{h}=-h^{2} \Delta+U^{h}, \quad D\left(H_{0}^{h}\right)=H^{2} \cap H_{0}^{1}(\Omega)
$$

and

$$
\tilde{H}_{0}^{h}=-\Delta+U, \quad D\left(\tilde{H}_{0}^{h}\right)=H^{2} \cap H_{0}^{1}\left(\Omega^{h}\right)
$$

respectively. The spectra of $H_{0}^{h}$ and $\tilde{H}_{0}^{h}$ are bounded from below by the norm $\|U\|_{L^{\infty}\left(\mathbb{R}^{d}\right)}$ and we can state

$$
\inf \left\{\varepsilon_{i}^{h}\right\}_{i \in \mathbb{N}} \geq-\|U\|_{L^{\infty}} .
$$

Due to the definition of the source term (1.5), $V^{h}$ and $\tilde{V}^{h}$ are generated by those energy levels $\varepsilon_{i}^{h}$ placed below the cut off $\varepsilon_{S}$ of the characteristic function $f$. In order to study the semiclassical behaviour of our system, we are interested into the spectral properties of the Hamiltonians

$$
H^{h}=-h^{2} \Delta+U^{h}+V^{h}, \quad D\left(H^{h}\right)=H^{2} \cap H_{0}^{1}(\Omega)
$$

and

$$
\tilde{H}^{h}=-\Delta+U+\tilde{V}^{h}, \quad D\left(\tilde{H}^{h}\right)=H^{2} \cap H_{0}^{1}\left(\Omega^{h}\right)
$$

in the spectral interval $\left[-\|U\|_{L^{\infty}}, \varepsilon_{S}\right)$, as $h \rightarrow 0$. In particular, a uniform bound for the number of eigenvalues $\varepsilon_{i}^{h} \in\left[-\|U\|_{L^{\infty}}, \varepsilon_{S}\right)$ as $h \rightarrow 0$ is required. Let us denote with $\left\{e_{i}^{h}\right\}_{i \in \mathbb{N}}$ the point spectrum of the unitarily equivalent Hamiltonians $H_{0}^{h}$ and $\tilde{H}_{0}^{h}$. As noticed above, the operators $H^{h}$ and $\tilde{H}^{h}$ are obtained as positive perturbations of $H_{0}^{h}$ and $\tilde{H}_{0}^{h}$ through the Poisson potentials $V^{h}$ and $\tilde{V}^{h}$ respectively. Then, the minimax principle implies that

$$
e_{i}^{h} \leq \varepsilon_{i}^{h} \quad \forall i \in \mathbb{N} .
$$

On the other hand, the eigenvalues $e_{i}^{h} \in\left[-\|U\|_{L^{\infty}}, \varepsilon_{S}\right)$ converge to eigenvalues of the operator $H_{0}=-\Delta+U$ on $\mathbb{R}^{d}$ as $h \rightarrow 0$. Such a standard result is a consequence of exponential decay estimates in classically forbidden region (the reader may refer to [8] for a general presentation and to Lemma 4.5 for a variation of those arguments in our nonlinear framework). Previous remarks lead to the following result. 
Lemma 2.1 There exists a finite natural $N_{0}$ such that $\forall h \in\left(0, h_{0}\right]$

$$
\begin{aligned}
& \#\left(\sigma\left(H_{0}^{h}\right) \cap\left[-\|U\|_{L^{\infty}}, \varepsilon_{S}\right)\right) \leq N_{0} \\
& \#\left(\sigma\left(H^{h}\right) \cap\left[-\|U\|_{L^{\infty}}, \varepsilon_{S}\right)\right) \leq N_{0}
\end{aligned}
$$

where $\sigma(H)$ denotes the spectrum of $H$.

Next we focus our attention on the Schrödinger-Poisson problem at the classical scale. To this concern we recall the variational formulation of this problem given in [12] for dimensions $d \leq 3$. Rephrasing the results of this work for our system, we can state that the solution to the equation $(1.4)(1.5)(1.7)$ is equivalent to the minimization problem

$$
\inf _{V \in H_{0}^{1}(\Omega)} J(V) ; \quad J(V)=\frac{1}{2} \int_{\Omega}|\nabla V(x)|^{2} d x+\operatorname{Tr}\left[F\left(H^{h}(V)\right)\right],
$$

where $F$ is the positive function

$$
F(x)=\int_{x}^{+\infty} f(s) d s
$$

while the Hamiltonian $H^{h}(V)$ is given by

$$
H^{h}(V)=-h^{2} \Delta+U^{h}+V, \quad D\left(H^{h}(V)\right)=H^{2} \cap H_{0}^{1}(\Omega) .
$$

Moreover, the function $J(V)$ is Fréchet- $C^{\infty}$ w.r.t. $V$, strictly convex and coercive, that is $\alpha$-convex, on $H_{0}^{1}(\Omega)$ and (2.11) admits a unique solution in this space. The following Proposition is a direct consequence of this result.

Proposition 2.2 The solutions to the Schrödinger-Poisson problem (1.4)(1.5)(1.7) are bounded in $H_{0}^{1}(\Omega)$ uniformly with respect to $h$.

Proof. From the variational formulation recalled above, the solution $V^{h}$ is the minimum of the convex map $J(V)$, therefore we have

$$
\frac{1}{2} \int_{\Omega}\left|\nabla V^{h}(x)\right|^{2} d x+\operatorname{Tr}\left[F\left(H^{h}\left(V^{h}\right)\right)\right] \leq J(0)=\operatorname{Tr}\left[F\left(H^{h}(0)\right)\right],
$$

where $H^{h}\left(V^{h}\right)$ simply coincides with the Hamiltonian $H^{h}$, while $H^{h}(0)$ can be identified with $H_{0}^{h}$ defined in (2.3). The relation

$$
\operatorname{Tr}\left[F\left(H^{h}\left(V^{h}\right)\right)\right]=\sum_{i \leq N_{0}} F\left(\varepsilon_{i}^{h}\right) \geq 0,
$$

with $N_{0}$ given in Lemma 2.1, implies

$$
\left\|V^{h}\right\|_{H_{0}^{1}(\Omega)}^{2} \leq 2 \operatorname{Tr}\left[F\left(H_{0}^{h}\right)\right] .
$$

The explicit expression of the r.h.s. here is

$$
\operatorname{Tr}\left[F\left(H_{0}^{h}\right)\right]=\sum_{i \leq N_{0}} F\left(e_{i}^{h}\right) .
$$

The result easily follows by combining (2.14) with the inequality

$$
\sum_{i \leq N_{0}} F\left(e_{i}^{h}\right) \leq N_{0} \sup _{x \in\left[-\|U\|_{L^{\infty}, \varepsilon_{S}}\right)} F<\infty .
$$

From equation (1.4) we have

$$
\left\|n\left[V^{h}\right]\right\|_{H^{-1}(\Omega)} \leq\left\|V^{h}\right\|_{H_{0}^{1}(\Omega)} .
$$

The forthcoming Corollary is a straightforward consequence of (2.15) and Proposition 2.2. 
Corollary 2.3 The charge density $n\left[V^{h}\right]$ is bounded in $H^{-1}(\Omega)$ uniformly with respect to $h$.

Next we use the assumption (1.16) and the estimates in Lemma A.1 of Appendix A to get uniform upper and lower bounds for the spectral points of $H^{h}$ as $h \rightarrow 0$.

Lemma 2.4 For $h_{0}$ small enough, the condition

$$
\varepsilon_{1}^{h}<\varepsilon_{S}
$$

holds for all $h \in\left(0, h_{0}\right]$.

Proof. We use a reductio ad absurdum argument. Let $\bar{h} \in\left(0, h_{0}\right]$ be such that $\varepsilon_{1}^{\bar{h}} \geq \varepsilon_{S}$. It follows from (1.2) and from the definition (1.5) that the corresponding charge density, $n\left[V^{\bar{h}}\right]$, and, then, the Poisson potential $V^{\bar{h}}$ are null in $\Omega$. In these conditions the Hamiltonians $H^{\bar{h}}$ and $H_{0}^{\bar{h}}$ coincide and we have

$$
\varepsilon_{1}^{\bar{h}}=e_{1}^{\bar{h}} \geq \varepsilon_{S} .
$$

On the other hand, we already noticed that $e_{1}^{h} \longrightarrow \inf \sigma\left(H_{0}\right)$ as $h \rightarrow 0$. Then from the assumption (1.16), the condition

$$
e_{1}^{\bar{h}}<\varepsilon_{S}
$$

definitely holds for $\bar{h} \rightarrow 0$, which is in contradiction with (2.17).

Theorem 2.5 The spectral points $\varepsilon_{i \leq N_{0}}^{h}$ fulfill the condition

$$
\lim \inf _{h \rightarrow 0} \varepsilon_{i}^{h} \geq \varepsilon_{S} .
$$

In particular, for $i=1$ we have

$$
\lim _{h \rightarrow 0} \varepsilon_{1}^{h}=\varepsilon_{S} .
$$

Proof. We work in the classical scale. Since $\left(\left|\Psi_{1}^{h}\right|^{2}\right)_{h \in\left(0, h_{0}\right]}$ is a family of probability measures it is weakly relatively compact in the set of bounded non negative Radon measures on $\Omega$ with total mass $\leq 1$. We first check that it converges to $\delta_{x_{0}}$ with the help of exponential decay estimates. From Lemma 2.4, it is known that: $\varepsilon_{1}^{h}<\varepsilon_{S}$ for all $h \in\left(0, h_{0}\right]$. Thus we can apply the estimates (A.5) to write

$$
\begin{aligned}
\left.\left|\int_{\Omega}\right| \Psi_{1}^{h}\right|^{2} \varphi d x \mid & \leq \int_{\operatorname{supp} \varphi}|\varphi| e^{-2 \phi / h}\left|e^{\phi / h} \Psi_{1}^{h}\right|^{2} d x \\
& \leq\|\varphi\|_{L^{\infty}}\left\|e^{\phi / h} \Psi_{1}^{h}\right\|_{L^{2}(\Omega)} \sup _{x \in \operatorname{supp} \varphi} e^{-2 \phi / h} \leq C\|\varphi\|_{L^{\infty}} \sup _{x \in \operatorname{supp} \varphi} e^{-\phi / h}
\end{aligned}
$$

for any $\varphi \in L^{\infty}(\Omega)$, while $\phi(x)$ is the Agmon distance from $x_{0}$ related to the potential $\left(U^{h}-\varepsilon_{S}\right)$ and defined by the relation (A.7) of the Appendix. When $\operatorname{supp} \varphi$ is a compact set in $\Omega \backslash\left\{x_{0}\right\}$, the inequality (A.15) says that there exists $c_{\varphi}>0$ such that

$$
\left.\left|\int_{\Omega}\right| \Psi_{1}^{h}\right|^{2} \varphi d x \mid \leq C\|\varphi\|_{L^{\infty}} e^{-c_{\varphi} / h}
$$

holds for $h>0$ small enough. By taking the limit as $h \rightarrow 0$, we get

$$
\left.\lim _{h \rightarrow 0}\left|\int_{\Omega}\right| \Psi_{1}^{h}\right|^{2} \varphi d x \mid=0
$$

for all continuous function $\varphi \in C^{0}(\bar{\Omega})$ with $\operatorname{supp} \varphi \subset \Omega \backslash\left\{x_{0}\right\}$. Hence the probability measure $\left|\Psi_{1}^{h}\right|^{2}$ converges in the narrow sense to $\delta_{x_{0}}$

$$
\int_{\Omega}\left|\Psi_{1}^{h}\right|^{2} \varphi d x \underset{h \rightarrow 0}{\longrightarrow} \varphi\left(x_{0}\right), \quad \forall \varphi \in C^{0}(\bar{\Omega}) .
$$


As a consequence of Corollary 2.3, the charge density: $n\left[V^{h}\right]=\sum_{i \leq N_{0}} f\left(\varepsilon_{i}^{h}\right)\left|\Psi_{i}^{h}\right|^{2}$ is uniformly bounded in $H^{-1}(\Omega)$ as $h \rightarrow 0$. Let $\varepsilon \in\left(0, \varepsilon_{0}\right]$ with $\varepsilon_{0}$ small enough, and consider the test function

$$
\varphi_{\varepsilon}(x)=\chi(x) \phi_{\varepsilon}\left(\frac{\left|x-x_{0}\right|}{2 R}\right), \quad x \in \Omega,
$$

where $R=\sup _{x \in \Omega}\left|x-x_{0}\right|, \chi \in C_{0}^{\infty}(\Omega)$ such that $\chi\left(x_{0}\right)=1$, and

$$
\phi_{\varepsilon}(u)=\left(\ln \frac{1}{\varepsilon}\right)^{\alpha} 1_{[0, \varepsilon]}(u)+\left(\ln \frac{1}{u}\right)^{\alpha} 1_{\left(\varepsilon, \frac{1}{2}\right]}(u),
$$

with $0<\alpha<\frac{d-1}{2}$. This function is continuous on $\bar{\Omega}$ and in dimension $d=2$ or 3 there exists $C_{\Omega}>0$ such that

$$
\forall \varepsilon \in\left(0, \varepsilon_{0}\right], \quad\left\|\varphi_{\varepsilon}\right\|_{H_{0}^{1}(\Omega)} \leq C_{\Omega} .
$$

We get

$$
f\left(\varepsilon_{1}^{h}\right) \int_{\Omega}\left|\Psi_{1}^{h}\right|^{2} \varphi_{\varepsilon} d x \leq\left\|n\left[V^{h}\right]\right\|_{H^{-1}(\Omega)}\left\|\varphi_{\varepsilon}\right\|_{H_{0}^{1}(\Omega)} \leq C_{\Omega}^{\prime} .
$$

Taking into account the boundary condition: $\varphi_{\varepsilon}\left(x_{0}\right)=\left(\ln \frac{1}{\varepsilon}\right)^{\alpha}$, the previous relation leads us to: $\lim \sup _{h \rightarrow 0} f\left(\varepsilon_{1}^{h}\right) \leq C_{\Omega}^{\prime}|\ln \varepsilon|^{-\alpha}$, for any $\varepsilon>0$, which implies

$$
\lim _{h \rightarrow 0} f\left(\varepsilon_{1}^{h}\right)=0 .
$$

Finally, making use of $\varepsilon_{i}^{h} \geq \varepsilon_{1}^{h}$ and $f^{\prime} \leq 0$, we get

$$
\lim _{h \rightarrow 0} f\left(\varepsilon_{i}^{h}\right)=0 .
$$

Remark 2.6 The informations given in the previous Theorem can be used to obtain some insight about the singularity of the Poisson potential $V^{h}$ in the limit $h \rightarrow 0$. Indeed, due to relation (2.23), the charge density converges to zero in the weak* topology of $H^{-1}(\Omega)$. Moreover, we know from Proposition 2.2 that the sequence of Poisson potentials $V^{h}$ is uniformly bounded in $H_{0}^{1}(\Omega)$ and, up to extraction, weakly convergent in this space. From the equation

$$
-\Delta V^{h}=n\left[V^{h}\right],
$$

and the continuity of $-\Delta$ on the space of distributions $\mathcal{D}^{\prime}$, we obtain that $V^{h}$ is weakly convergent to zero in $H_{0}^{1}(\Omega)$. Fixing $p \in[1,6)$ in dimension $d=3$ or $p \in[1,+\infty)$ in dimension $d=2$, the previous result and the compact injection $H^{1}(\Omega) \hookrightarrow \hookrightarrow L^{p}(\Omega)$ (e.g. in [5]), give the convergence $V^{h} \rightarrow 0$ in the strong $L^{p}$-norm sense. However, the asymptotic condition (2.20) implies that, in the limit $h \rightarrow 0$, the Poisson potential produces a non null spectral perturbation of the limit Hamiltonian - given by $H_{0}(1.14)$ at the quantum scale. For this reason we expect that $V^{h} \nrightarrow 0$ in $L^{\infty}(\Omega)$.

Furthermore, we have some strong convergence for the density: using the normalization (1.12) of the eigenfunctions, we can write

$$
\left\|n\left[V^{h}\right]\right\|_{L^{1}(\Omega)} \leq \sum_{i \leq N_{0}} f\left(\varepsilon_{i}^{h}\right)
$$

and, applying (2.23), we obtain that $n\left[V^{h}\right] \underset{h \rightarrow 0}{\longrightarrow} 0$ strongly in $L^{1}(\Omega)$ (and therefore strongly in the space of bounded measures $\mathcal{M}_{b}(\Omega)$ ).

We now return to the quantum scale setting. Let us denote with $A_{i}^{h}$

$$
A_{i}^{h}=h^{2-d} f\left(\varepsilon_{i}^{h}\right) .
$$


With this notations, the charge density at the quantum scale is described by

$$
\rho^{h}=\sum_{i \leq N_{0}} A_{i}^{h}\left|\tilde{\Psi}_{i}^{h}\right|^{2}, \quad h \in\left(0, h_{0}\right]
$$

and our system writes as

$$
\left\{\begin{array}{l}
\left(-\Delta+U+\tilde{V}^{h}\right) \tilde{\Psi}_{i}^{h}=\varepsilon_{i}^{h} \tilde{\Psi}_{i}^{h}, \quad \text { in } \Omega^{h} \\
-\Delta \tilde{V}^{h}=\rho^{h}, \quad \text { in } \Omega^{h} \\
\left.\tilde{V}^{h}\right|_{\partial \Omega^{h}}=0,\left.\quad \tilde{\Psi}_{i}^{h}\right|_{\partial \Omega^{h}}=0
\end{array} \quad ; \quad h \in\left(0, h_{0}\right] .\right.
$$

Lemma 2.7 The coefficients $A_{i \leq N_{0}}^{h}$ show the following properties:

- In dimension $d=3$ : the set $\left\{A_{i \leq N_{0}}^{h}, h \in\left(0, h_{0}\right]\right\}$ is uniformly bounded w.r.t. $h$.

- In dimension $d=2$ : the set $\left\{A_{i \leq N_{0}}^{h} \ln \frac{1}{h}, h \in\left(0, h_{0}\right]\right\}$ is uniformly bounded w.r.t. $h$. where $\left(0, h_{0}\right]$ is a suitable right neighbourhood of the origin.

Proof. First notice that, if $\varepsilon_{i}^{h} \geq \varepsilon_{S}$, we have: $A_{i}^{h}=0$ and the statement is trivial. Thus, we can assume in what follows: $\varepsilon_{i}^{h}<\varepsilon_{S}$. This allows us to apply the estimates (A.17) and (A.18) to the eigenvectors $\tilde{\Psi}_{i \leq N_{0}}^{h}$ in the limit $h \rightarrow 0$. Let $B_{r}$ denotes the ball of radius $r$ centered in the origin of $\mathbb{R}^{d}$; for $h \rightarrow 0$, the condition: $B_{r} \subset \Omega^{h}$ definitely holds, and we can write

$$
1=\left\|\tilde{\Psi}_{i}^{h}\right\|_{L^{2}\left(\Omega^{h}\right)}^{2}=\int_{B_{r}}\left|\tilde{\Psi}_{i}^{h}\right|^{2} d x+\int_{\Omega^{h} \backslash B_{r}} e^{-2 c_{0}|x|}\left|e^{c_{0}|x|} \tilde{\Psi}_{i}^{h}\right|^{2} d x .
$$

Corollary A.4 gives an estimate for the r.h.s. of this expression

$$
\int_{B_{r}}\left|\tilde{\Psi}_{i}^{h}\right|^{2} d x+\int_{\Omega^{h} \backslash B_{r}} e^{-2 c_{0}|x|}\left|e^{c_{0}|x|} \tilde{\Psi}_{i}^{h}\right|^{2} d x \leq \int_{B_{r}}\left|\tilde{\Psi}_{i}^{h}\right|^{2} d x+C e^{-2 c_{0} r} .
$$

Fixing $r$ such that: $C e^{-2 c_{0} r} \leq \frac{1}{2}$, which is always possible for $h_{0}$ close enough to the origin, the previous relations implies

$$
\int_{B_{r}}\left|\tilde{\Psi}_{i}^{h}\right|^{2} d x \geq \frac{1}{2}, \quad \forall h \in\left(0, h_{0}\right] .
$$

This relation can be used to get an estimate for the potential $\tilde{V}^{h}$ inside $B_{r}$. Indeed, from Lemma 2.4 , we have

$$
\varepsilon_{S}>\varepsilon_{1}^{h}, \quad \forall h \in\left(0, h_{0}\right] .
$$

Moreover, it follows from the relation

$$
\varepsilon_{1}^{h}=\left(\tilde{\Psi}_{1}^{h}, \tilde{H}^{h} \tilde{\Psi}_{1}^{h}\right)_{L^{2}\left(\Omega^{h}\right)},
$$

that

$$
\varepsilon_{S}>\varepsilon_{1}^{h} \geq-\|U\|_{L^{\infty}}+\left(\inf _{x \in B_{r}} \tilde{V}^{h}\right) \int_{B_{r}}\left|\tilde{\Psi}_{i}^{h}\right|^{2} d x \geq-\|U\|_{L^{\infty}}+\frac{1}{2} \inf _{x \in B_{r}} \tilde{V}^{h}
$$

which implies

$$
\inf _{x \in B_{r}} \tilde{V}^{h} \leq C
$$

with $C>0$.

Let us denote with $I_{B_{r}}$ the characteristic function of the ball $B_{r}$ and with $W^{h}$ the solution to the following problem

$$
\left\{\begin{array}{l}
-\Delta W^{h}=\rho_{r} \\
\left.W^{h}\right|_{\partial \Omega^{h}}=0
\end{array} \text { in } \Omega^{h}\right.
$$


with

$$
\rho_{r}=I_{B_{r}} \sum_{i \leq N_{0}} A_{i}^{h}\left|\tilde{\Psi}_{i}^{h}(x)\right|^{2}
$$

Notice that

$$
\rho^{h} \geq \rho_{r} \text { on } \Omega^{h} .
$$

Therefore, by applying the maximum principle to the equation

$$
\left\{\begin{array}{l}
-\Delta\left(\tilde{V}^{h}-W^{h}\right)=\rho^{h}-\rho_{r} \quad \text { in } \Omega^{h}, \\
\left.\left(\tilde{V}^{h}-W^{h}\right)\right|_{\partial \Omega^{h}}=0,
\end{array}\right.
$$

we get $\tilde{V}^{h} \geq W^{h}$ in $\Omega^{h}$. The strategy of our proof is to show that the lower bound on $B_{r}$ for $W^{h}$ depends on the sum $\sum_{i \leq N_{0}} A_{i}^{h}$ in the 3-D case, or $\ln \frac{1}{h} \sum_{i \leq N_{0}} A_{i}^{h}$ in the 2-D case. We will consider the 3 -D and the 2-D cases separately.

Let $d=3$. In order to obtain a lower bound for the function $W^{h}$, we compare $W^{h}$ with $G * \rho_{r}$, where $G=\frac{1}{4 \pi|x|}$ is the Green kernel of the Laplacian in $\mathbb{R}^{3}$ while ' $*$ ' denotes the convolution operation. The difference $W^{h}-G * \rho_{r}$ solves the problem

$$
\left\{\begin{array}{l}
-\Delta u=0 \text { in } \Omega^{h} \\
\left.u\right|_{\partial \Omega^{h}}=-G * \rho_{r}
\end{array}\right.
$$

Recalling that $G * \rho_{r}$ is a positive function, the maximum principle applied to (2.29) leads to

$$
\left(W^{h}-G * \rho_{r}\right) \geq-\sup _{x \in \partial \Omega^{h}}\left(G * \rho_{r}\right) \quad \text { in } \Omega^{h},
$$

from which we have

$$
W^{h} \geq G * \rho_{r}-\sup _{x \in \partial \Omega^{h}}\left(G * \rho_{r}\right) \quad \text { in } \Omega^{h} .
$$

- For $x \in \partial \Omega^{h}$ we have: $|x| \sim \mathcal{O}\left(\frac{1}{h}\right)$ as $h \rightarrow 0$. Take $R=d\left(B\left(x_{0}, R\right), \partial \Omega\right)$ in the classical scale. Then at the quantum scale, the inequality

$$
|x-y| \geq \frac{R}{h}, \quad \forall y \in B_{r},
$$

holds for any $h \in\left(0, h_{0}\right]$. Using $(2.31)$ we get

$$
\begin{aligned}
\left.G * \rho_{r}\right|_{\partial \Omega^{h}} & =\left.\frac{1}{4 \pi} \sum_{i \leq N_{0}} A_{i}^{h} \int_{B_{r}} \frac{1}{|x-y|}\left|\tilde{\Psi}_{i}^{h}(y)\right|^{2} d y\right|_{\partial \Omega^{h}} \\
& \leq \frac{h}{4 \pi R} \sum_{i \leq N_{0}} A_{i}^{h} \int_{B_{r}}\left|\tilde{\Psi}_{i}^{h}(y)\right|^{2} d y \leq \frac{h}{4 \pi R}\left(\sum_{i \leq N_{0}} A_{i}^{h}\right) .
\end{aligned}
$$

- For $x, y \in B_{r} \rightarrow \frac{1}{|x-y|} \geq \frac{1}{2 r}$. From this condition and (2.27) it follows

$$
\left.G * \rho_{r}\right|_{B_{r}} \geq \frac{1}{8 \pi r} \sum_{i \leq N_{0}} A_{i}^{h} \int_{B_{r}}\left|\tilde{\Psi}_{i}^{h}(y)\right|^{2} d y \geq \frac{1}{16 \pi r}\left(\sum_{i \leq N_{0}} A_{i}^{h}\right) .
$$

Making use of (2.30), (2.32) and (2.33), and assuming $h \rightarrow 0$, we get

$$
\begin{aligned}
\left.W^{h}\right|_{B_{r}} & \geq \frac{1}{16 \pi r}\left(\sum_{i \leq N_{0}} A_{i}^{h}\right)-\frac{h}{4 \pi R}\left(\sum_{i \leq N_{0}} A_{i}^{h}\right)=\frac{1}{16 \pi r}\left(\sum_{i \leq N_{0}} A_{i}^{h}\right)\left(1-\frac{4 r}{R} h\right) \\
& \geq \frac{1}{32 \pi r}\left(\sum_{i \leq N_{0}} A_{i}^{h}\right) .
\end{aligned}
$$


Recalling that $\tilde{V}^{h} \geq W^{h}$ on $\Omega^{h}$, it follows from the last inequality that

$$
\left.\tilde{V}^{h}\right|_{B_{r}} \geq \frac{1}{32 \pi r}\left(\sum_{i \leq N_{0}} A_{i}^{h}\right), \quad \forall h \in\left(0, h_{0}\right] .
$$

Combining this condition with (2.28) we get a uniform bound for $\sum_{i \leq N_{0}} A_{i}^{h}$ as $h \in\left(0, h_{0}\right]$. This concludes the proof in the 3 -D case.

The 2-D case follows essentially the same line. Nevertheless, it is worthwhile to notice that the Green kernel of the 2-D Laplacian, $G(x)=-\frac{1}{2 \pi} \ln |x|$, does not have a fixed sign. Therefore, relation (2.30) will be replaced by

$$
W^{h} \geq G * \rho_{r}+\inf _{x \in \Omega^{h}}\left(-G * \rho_{r}\right) \text { in } \Omega^{h},
$$

while (2.32) and (2.33) respectively by

$$
\begin{aligned}
-\left.G * \rho_{r}\right|_{\partial \Omega^{h}} & =\left.\frac{1}{2 \pi} \sum_{i \leq N_{0}} A_{i}^{h} \int_{B_{r}} \ln |x-y|\left|\tilde{\Psi}_{i}^{h}(y)\right|^{2} d y\right|_{\partial \Omega^{h}} \geq \frac{1}{2 \pi} \ln \frac{R}{h} \sum_{i \leq N_{0}} A_{i}^{h} \int_{B_{r}}\left|\tilde{\Psi}_{i}^{h}(y)\right|^{2} d y \\
& \geq \frac{1}{4 \pi}\left(\sum_{i \leq N_{0}} A_{i}^{h}\right)\left(\ln R+\ln \frac{1}{h}\right),
\end{aligned}
$$

and

$$
\left.G * \rho_{r}\right|_{B_{r}} \geq \frac{1}{2 \pi} \ln \frac{1}{2 r} \sum_{i \leq N_{0}} A_{i}^{h} \int_{B_{r}}\left|\tilde{\Psi}_{i}^{h}(y)\right|^{2} d y \geq \frac{1}{4 \pi}\left(\sum_{i \leq N_{0}} A_{i}^{h}\right) \ln \frac{1}{2 r} .
$$

Remark 2.8 The previous result confirms the relation (2.23) obtained in the proof of Theorem 2.5. Moreover, it allows to establish a precise asymptotic order for $f\left(\varepsilon_{i \leq N_{0}}^{h}\right)$ when $h \rightarrow 0$

- In dimension 3: $f\left(\varepsilon_{i \leq N_{0}}^{h}\right)=\mathcal{O}(h)$.

- In dimension 2: $f\left(\varepsilon_{i \leq N_{0}}^{h}\right)=\mathcal{O}\left(\left(\ln \frac{1}{h}\right)^{-1}\right)$.

The next Lemma characterizes the compactness of the family $\tilde{\Psi}_{i \leq N_{0}}^{h}$ as $h \rightarrow 0$. In what follows $\varepsilon$ denotes a negative constant and $\mathbb{I}_{\Omega^{h}, \varepsilon}$ denotes the characteristic function

$$
\mathbb{I}_{\Omega^{h}, \varepsilon}(x, \lambda)= \begin{cases}1 & \text { for } x \in \Omega^{h} \text { and } \lambda<\varepsilon \\ 0 & \text { otherwise }\end{cases}
$$

Lemma 2.9 For $i \leq N_{0}, \varepsilon \in\left(-\|U\|_{L^{\infty}}, 0\right)$ and $h \in\left(0, h_{0}\right]$, with $h_{0}>0$ small enough, the following properties hold:

- In dimension $d=3$, the family $\left(\mathbb{I}_{\Omega^{h}, \varepsilon}\left(\cdot, \varepsilon_{i}^{h}\right) \tilde{\Psi}_{i}^{h}\right)_{h \in\left(0, h_{0}\right]}$ is relatively compact in $L^{p}\left(\mathbb{R}^{3}\right)$ with $p \in[1,6)$.

- In dimension $d=2$, the family $\left(\mathbb{I}_{\Omega^{h}, \varepsilon}\left(\cdot, \varepsilon_{i}^{h}\right) \tilde{\Psi}_{i}^{h}\right)_{h \in\left(0, h_{0}\right]}$ is relatively compact in $L^{p}\left(\mathbb{R}^{2}\right)$ with $p \in[1,+\infty)$.

- Hence in both cases $\left(\left|\mathbb{I}_{\Omega^{h}, \varepsilon}\left(\cdot, \varepsilon_{i}^{h}\right) \tilde{\Psi}_{i}^{h}\right|^{2}\right)_{h \in\left(0, h_{0}\right]}$ is relatively compact in $L^{1} \cap L^{2}\left(\mathbb{R}^{d}\right)$. 
Proof. Fix $p$ as follows: $p \in[1,6)$ in dimension $d=3, p \in[1,+\infty)$ in dimension $d=2$. Due to definition (2.36), we can restrict our investigation to the case $\varepsilon_{i}^{h}<\varepsilon$. From Corollary A.4, we have

$$
\left\|\tilde{\Psi}_{i}^{h}\right\|_{L^{p}\left(\Omega^{h}\right)} \leq\left\|e^{c_{0}|x|} \tilde{\Psi}_{i}^{h}\right\|_{L^{p}\left(\Omega^{h}\right)} \leq C, \quad \forall h \in\left(0, h_{0}\right] .
$$

Thus, the family $\left(\mathbb{I}_{\Omega^{h}, \varepsilon}\left(\cdot, \varepsilon_{i}^{h}\right) \tilde{\Psi}_{i}^{h}\right)_{h \in\left(0, h_{0}\right]}$ is uniformly bounded in $L^{p}\left(\mathbb{R}^{d}\right)$. Moreover, for any bounded domain $B \subset \mathbb{R}^{d}$, it follows, again from Corollary A.4, that

$$
\begin{aligned}
\left\|\mathbb{I}_{\Omega^{h}, \varepsilon}\left(\cdot, \varepsilon_{i}^{h}\right) \tilde{\Psi}_{i}^{h}\right\|_{H^{1}(B)} & \leq\left\|\tilde{\Psi}_{i}^{h}\right\|_{H^{1}\left(\Omega^{h}\right)} \\
& \leq\left\|e^{c_{0}|\cdot|} \nabla \tilde{\Psi}_{i}^{h}\right\|_{L^{2}\left(\Omega^{h}\right)}+\left\|e^{c_{0}|\cdot|} \tilde{\Psi}_{i}^{h}\right\|_{L^{2}\left(\Omega^{h}\right)} \leq C
\end{aligned}
$$

for all $h \in\left(0, h_{0}\right]$. By the compactness of the injection of $H^{1}(B) \hookrightarrow L^{p}(B),\left\{\mathbb{I}_{\Omega^{h}, \varepsilon}\left(\cdot, \varepsilon_{i}^{h}\right) \tilde{\Psi}_{i}^{h}\right\}_{h \in\left(0, h_{0}\right]}$ is relatively compact in $L^{p}(B)$. Next, consider the $L^{p}$-norm of $\mathbb{I}_{\Omega^{h}, \varepsilon}\left(\cdot, \varepsilon_{i}^{h}\right) \tilde{\Psi}_{i}^{h}$ on $\mathbb{R}^{d} \backslash B$

$$
\begin{aligned}
\left\|\mathbb{I}_{\Omega^{h}, \varepsilon}\left(\cdot, \varepsilon_{i}^{h}\right) \tilde{\Psi}_{i}^{h}\right\|_{L^{p}\left(\mathbb{R}^{d} \backslash B\right)}^{p} & =\int_{\Omega^{h} \backslash B} e^{-p c_{0}|x|}\left|e^{c_{0}|x|} \tilde{\Psi}_{i}^{h}\right|^{p} d x \\
& \leq\left(\int_{\Omega^{h}}\left|e^{c_{0}|x|} \tilde{\Psi}_{i}^{h}\right|^{p} d x\right) \sup _{x \in \mathbb{R}^{d} \backslash B} e^{-p c_{0}|x|} \leq C^{\prime} \sup _{x \in \mathbb{R}^{d} \backslash B} e^{-p c_{0}|x|} .
\end{aligned}
$$

For any $\eta>0$, there exists a bounded domain $B_{\eta}$ such that

$$
\forall h \in\left(0, h_{0}\right], \quad\left\|\mathbb{I}_{\Omega^{h}, \varepsilon}\left(\cdot, \varepsilon_{i}^{h}\right) \tilde{\Psi}_{i}^{h}\right\|_{L^{p}\left(\mathbb{R}^{d} \backslash B_{\eta}\right)}<\eta
$$

This and the relative compactness on any bounded $B$ due to Sobolev imbeddings provide the relative compactness on the whole space $\mathbb{R}^{d}$ (see Corollary IV.26 in [5]).

The third point follows from the Hölder inequality

$$
\left\||f|^{2}-|g|^{2}\right\|_{L^{p}\left(\mathbb{R}^{d}\right)} \leq\|f-g\|_{L^{2 p}\left(\mathbb{R}^{d}\right)}\left(\|f\|_{L^{2 p}\left(\mathbb{R}^{d}\right)}+\|g\|_{L^{2 p}\left(\mathbb{R}^{d}\right)}\right)
$$

with $p=1$ or $p=2$.

\section{The asymptotic problem in the $3-\mathrm{D}$ case}

\subsection{Asymptotic behaviour of the Poisson potential and the limit Poisson problem}

As already noticed in Remark 2.6, the role played by the Poisson potential at the classical scale, $V^{h}$, presents an ambiguous interpretation. Indeed, it strongly converges to zero in $L^{p}, p<6$, as $h \rightarrow 0$, producing, at the same time, a non null spectral perturbation of the limit Hamiltonian, corresponding to the spectral shift: $\varepsilon_{S}-e_{1}$ (see the condition (2.20)). This ambiguity disappear when the problem is considered at the quantum scale. In this Section we will show that, in the 3-D case, the Poisson potential at the quantum scale has a non trivial asymptotic behaviour described by a limit equation of Schrödinger-Poisson kind. The strategy of our proof consists in exploiting the boundedness of the coefficients $A_{i}^{h}$ and the relative compactness properties of the eigenvectors $\tilde{\Psi}_{i}^{h}$, with $\varepsilon_{i}^{h}<0$, to extract a converging sequence of charge densities. Then, as an intermediate result, a limit Schrödinger-Poisson equation is obtained, modulo an extraction, by using standard estimates (see (3.3) and (3.4) below) and the elliptic regularity of the limit Hamiltonian. In the end, a uniqueness result for this asymptotic problem will ensure the convergence of the whole family as $h \rightarrow 0$. The intermediate arguments will often be written with the notation

$$
\lim _{\substack{h \rightarrow 0 \\ h \in D}} g(h)=\gamma
$$


where $D$ denotes a well chosen, countable or not, subset $D \subset\left(0, h_{0}\right]$ such that $0 \in \bar{D}$. For example the relative compactness stated in Lemma 2.7 and Lemma 2.9 can be used as follows: out of any infinite subset $S \subset\left(0, h_{0}\right]$ with $0 \in \bar{S}$, such a countable subset $D$ can be extracted so that

$$
\begin{aligned}
& \lim _{\substack{h \rightarrow 0 \\
h \in D}}\left\|1_{\Omega^{h}} \rho^{h}-\rho\right\|_{L^{1} \cap L^{2}\left(\mathbb{R}^{3}\right)}=0, \\
& \text { with } \rho=\sum_{i \leq N_{0}} A_{i}\left|\chi_{i}\right|^{2}, \quad A_{i}=\lim _{\substack{h \rightarrow 0 \\
h \in D}} A_{i}^{h} \geq 0 \quad \text { and } \quad \lim _{\substack{h \rightarrow 0 \\
h \in D}}\left\|\mathbb{I}_{\Omega^{h}, \varepsilon}\left(\cdot, \varepsilon_{i}^{h}\right) \tilde{\Psi}_{i}^{h}-\chi_{i}\right\|_{L^{2} \cap L^{4}\left(\mathbb{R}^{3}\right)}=0 .
\end{aligned}
$$

where $\varepsilon$ is a constant in $\left[\varepsilon_{S}, 0\right)$.

Proposition 3.1 Let $G$ be the Green function of the Laplace operator in $\mathbb{R}^{3}$. For a set $D$ such that the conditions (3.2)-(3.1) are verified, the potential at the quantum scale $1_{\Omega^{h}} \tilde{V}^{h}$ satisfies

$$
\lim _{\substack{h \rightarrow 0 \\ h \in D}}\left\|1_{\Omega^{h}} \tilde{V}^{h}-G * \varrho\right\|_{L^{\infty}\left(\mathbb{R}^{3}\right)}=0 .
$$

Proof. We start recalling a standard estimate. Let $f \in L^{1} \cap L^{2}\left(\mathbb{R}^{3}\right)$ and consider the convolution $G * f$, whose Fourier Transform is

$$
\mathcal{F}(G * f)(k)=\frac{\mathcal{F} f(k)}{k^{2}}
$$

$\mathcal{F} f$ denoting the Transform of $f$. From the Young-Housdorff inequality, we know that $\mathcal{F} f \in$ $L^{2} \cap L^{\infty}\left(\mathbb{R}^{3}\right)$, which implies, using standard estimates, that

$$
\left\|\frac{\mathcal{F} f(k)}{k^{2}}\right\|_{L^{1}\left(\mathbb{R}^{3}\right)} \leq C\left(\|f\|_{L^{1}\left(\mathbb{R}^{3}\right)}+\|f\|_{L^{2}\left(\mathbb{R}^{3}\right)}\right) .
$$

Then, we get

$$
\|G * f\|_{L^{\infty}\left(\mathbb{R}^{3}\right)} \leq C\left(\|f\|_{L^{1}\left(\mathbb{R}^{3}\right)}+\|f\|_{L^{2}\left(\mathbb{R}^{3}\right)}\right) .
$$

Moreover, from the above condition $\frac{\mathcal{F} f(k)}{k^{2}} \in L^{1}\left(\mathbb{R}^{3}\right)$ and the Riemann-Lebesgue Lemma, we also obtain that the convolution $G * f$ belongs to the space $C_{\infty}\left(\mathbb{R}^{3}\right)$ of continuous functions vanishing at $\infty$

$$
G * f \in C_{\infty}\left(\mathbb{R}^{3}\right) .
$$

The Poisson potential can be expressed by the action of the inverse Dirichlet-Laplacian on $\rho^{h}$ : $\tilde{V}^{h}=\left(-\Delta_{\Omega^{h}}^{D}\right)^{-1} \rho^{h}$, and the difference $\tilde{V}^{h}-G * \rho$ is bounded in $\Omega^{h}$ by

$$
\begin{aligned}
& \left\|\tilde{V}^{h}-G * \rho\right\|_{L^{\infty}\left(\Omega^{h}\right)} \leq\left\|G * \rho-\left(-\Delta_{\Omega^{h}}^{D}\right)^{-1} \rho\right\|_{L^{\infty}\left(\Omega^{h}\right)}+ \\
& +\left\|G *\left(\rho-1_{\Omega^{h}} \rho^{h}\right)-\left(-\Delta_{\Omega^{h}}^{D}\right)^{-1}\left(\rho-\rho^{h}\right)\right\|_{L^{\infty}\left(\Omega^{h}\right)}+\left\|G *\left(\rho-1_{\Omega^{h}} \rho^{h}\right)\right\|_{L^{\infty}\left(\Omega^{h}\right)} .
\end{aligned}
$$

The maximum principle, applied to the equation

$$
\left\{\begin{array}{l}
-\Delta u=0 \quad \text { in } \Omega^{h} \\
\left.u\right|_{\partial \Omega^{h}}=\left.G * f\right|_{\partial \Omega^{h}}
\end{array}\right.
$$

with $f \in L^{1} \cap L^{2}\left(\mathbb{R}^{3}\right)$, leads us to the estimate

$$
\|u\|_{L^{\infty}\left(\Omega^{h}\right)} \leq \sup _{x \in \partial \Omega^{h}}|G * f| .
$$


If $u$ is identified with the functions $G * \rho-\left(-\Delta_{\Omega^{h}}^{D}\right)^{-1} \rho$ and $G *\left(\rho-1_{\Omega^{h}} \rho^{h}\right)-\left(-\Delta_{\Omega^{h}}^{D}\right)^{-1}\left(\rho-\rho^{h}\right)$ appearing at the r.h.s of (3.5), the previous estimates gives

$$
\begin{aligned}
\left\|\tilde{V}^{h}-G * \rho\right\|_{L^{\infty}\left(\Omega^{h}\right)} & \leq \sup _{x \in \partial \Omega^{h}}|G * \rho|+\sup _{x \in \partial \Omega^{h}}\left|G *\left(\rho-1_{\Omega^{h}} \rho^{h}\right)\right|+\left\|G *\left(\rho-1_{\Omega^{h}} \rho^{h}\right)\right\|_{L^{\infty}\left(\Omega^{h}\right)} \\
& \leq \sup _{x \in \partial \Omega^{h}}|G * \rho|+2\left\|G *\left(\rho-1_{\Omega^{h}} \rho^{h}\right)\right\|_{L^{\infty}\left(\mathbb{R}^{3}\right)} .
\end{aligned}
$$

Applying the properties (3.4) to $G * \rho$ and (3.3) to $G *\left(\rho-1_{\Omega^{h}} \rho^{h}\right)$, it follows from the conditions $\Omega^{h} \rightarrow \mathbb{R}^{3}$ and (3.1) that

$$
\lim _{\substack{h \rightarrow 0 \\ h \in D}}\left\|\tilde{V}^{h}-G * \rho\right\|_{L^{\infty}\left(\Omega^{h}\right)}=0 .
$$

In the exterior domain $\mathbb{R}^{3} \backslash \Omega^{h}$, we have

$$
\left\|1_{\Omega^{h}} \tilde{V}^{h}-G * \rho\right\|_{L^{\infty}\left(\mathbb{R}^{3} \backslash \Omega^{h}\right)}=\|G * \rho\|_{L^{\infty}\left(\mathbb{R}^{3} \backslash \Omega^{h}\right)}^{\underset{h \rightarrow 0}{\longrightarrow} 0},
$$

where (3.4) has been once more implemented.

From (3.6) and (3.7) it finally follows that

$$
\left\|1_{\Omega^{h}} \tilde{V}^{h}-G * \rho\right\|_{L^{\infty}\left(\mathbb{R}^{3}\right)}=\max \left\{\|G * \rho\|_{L^{\infty}\left(\mathbb{R}^{3} \backslash \Omega^{h}\right)},\left\|\tilde{V}^{h}-G * \rho\right\|_{L^{\infty}\left(\Omega^{h}\right)}\right\} \underset{h \rightarrow 0, h \in D}{\longrightarrow} 0
$$

which concludes the proof.

Concerning the problem at the classical scale, we can actually strengthen the result referred in Remark 2.6.

Corollary 3.2 Under the assumptions of Proposition 3.1, the solution of the Schrödinger-Poisson problem at the classical scale, (1.4)(1.5)(1.7), strongly converges to 0 in $H_{0}^{1}(\Omega)$ as $h \rightarrow 0$ with

$$
\left\|V^{h}\right\|_{H_{0}^{1}(\Omega)}=\mathcal{O}\left(h^{\frac{1}{2}}\right) .
$$

Proof. The Poisson potentials at the classical and quantum scales are related by the change of variables: $\Omega \rightarrow \Omega^{h}$ and the relation (1.11). In 3D, we have

$$
\left\|V^{h}\right\|_{H_{0}^{1}(\Omega)}=h^{1 / 2}\left\|\tilde{V}^{h}\right\|_{H_{0}^{1}\left(\Omega^{h}\right)} .
$$

Projecting the Poisson equation for $\tilde{V}^{h}$ (the second one in (2.26)) over $\tilde{V}^{h}$ itself, the norm $\left\|\tilde{V}^{h}\right\|_{H_{0}^{1}\left(\Omega^{h}\right)}^{2}$ is estimated by

$$
\left\|\tilde{V}^{h}\right\|_{H_{0}^{1}\left(\Omega^{h}\right)}^{2}=\sum_{i \leq N_{0}} A_{i}^{h} \int_{\Omega^{h}}\left|\tilde{\Psi}_{i}^{h}\right|^{2} \tilde{V}^{h} d x \leq\left\|\tilde{V}^{h}\right\|_{L^{\infty}\left(\mathbb{R}^{3}\right)} \sum_{i \leq N_{0}} A_{i}^{h} .
$$

As it follows from Lemma 2.7 and Proposition 3.1, the coefficients $A_{i}^{h}$ are bounded and the family of potentials $\left(1_{\Omega^{h}} \tilde{V}^{h}\right)_{h \in D}$ converges in $L^{\infty}\left(\mathbb{R}^{3}\right)$ as $h \rightarrow 0$. Therefore we have

$$
\left\|\tilde{V}^{h}\right\|_{H_{0}^{1}\left(\Omega^{h}\right)} \leq C, \quad h \in D
$$

and, combining (3.8) and (3.9),

$$
\left\|V^{h}\right\|_{H_{0}^{1}(\Omega)}=\mathcal{O}\left(h^{\frac{1}{2}}\right), \quad h \in D .
$$

This concludes the proof.

Next we investigate the limit shape of the family of Schrödinger-Poisson problems when $h$ belongs to a subset $D$ verifying the conditions (3.2) and (3.1). 
Proposition 3.3 Under the assumptions of Proposition 3.1, the following limits hold

$$
\begin{aligned}
& \lim _{h \rightarrow 0} \inf \varepsilon_{i}^{h}>\varepsilon_{S} \quad \text { for } i>1, \\
& \lim _{\substack{h \rightarrow 0 \\
h \in D}} A_{1}^{h}=A, \quad \lim _{\substack{h \rightarrow 0 \\
h \in D}}\left\|1_{\Omega^{h}} \tilde{\Psi}_{1}^{h}-\chi\right\|_{L^{2} \cap L^{4}\left(\mathbb{R}^{3}\right)}=0, \quad \lim _{\substack{h \rightarrow 0 \\
h \in D}}\left\|1_{\Omega^{h}} \tilde{V}^{h}-G * A|\chi|^{2}\right\|_{L^{\infty}\left(\mathbb{R}^{3}\right)}=0,
\end{aligned}
$$

where $A$ and $\chi$ solve the problem

$$
\left\{\begin{array}{l}
{[-\Delta+U+W] \chi=\varepsilon_{S} \chi, \quad\|\chi\|_{L^{2}\left(\mathbb{R}^{3}\right)}=1} \\
\varepsilon_{S}=\inf \sigma(-\Delta+U+W) \\
-\Delta W=A|\chi|^{2}
\end{array}\right.
$$

Proof. Let $N_{1}$ be defined by

$$
N_{1}=\max \left\{i \mid \lim _{h \rightarrow 0} \inf \varepsilon_{i}^{h}=\varepsilon_{S}\right\} .
$$

Due to the result of Theorem 2.5, we know that $N_{1} \geq 1$. Rephrasing the argument used at the beginning of this Section, it is possible to find a set $D \subset\left(0, h_{0}\right], 0 \in \bar{D}$, such that the conditions (3.1) - (3.2) are verified and

$$
\lim _{\substack{h \rightarrow 0 \\ h \in D}} \varepsilon_{i \leq N_{1}}^{h}=\varepsilon_{S}
$$

In what follows, the constant $\varepsilon$, appearing in (3.2), is strictly greater that $\varepsilon_{S}$. If we choose $h_{0}$ small enough, this condition and the previous limit imply: $\varepsilon_{i}^{h}<\varepsilon$, for all $h \in D$. Set $i \leq N_{1}$, $\varphi \in C_{0}^{\infty}\left(\mathbb{R}^{3}\right)$. According to (3.14), and to the results of Lemma 2.9 and Proposition 3.1, the following limit holds

$$
\lim _{\substack{h \rightarrow 0 \\ h \in D}}\left(\left[-\Delta+U+\tilde{V}^{h}-\varepsilon_{i}^{h}\right] \varphi, 1_{\Omega^{h}} \tilde{\Psi}_{i}^{h}\right)_{L^{2}\left(\mathbb{R}^{3}\right)}=\left(\left[-\Delta+U+G * \rho-\varepsilon_{S}\right] \varphi, \chi_{i}\right)_{L^{2}\left(\mathbb{R}^{3}\right)},
$$

with

$$
\rho=\sum_{i \leq N_{0}} A_{i}\left|\chi_{i}\right|^{2}, \quad A_{i}=\lim _{\substack{h \rightarrow 0 \\ h \in D}} A_{i}^{h} \geq 0 \quad \text { and } \quad \lim _{\substack{h \rightarrow 0 \\ h \in D}}\left\|\mathbb{I}_{\Omega^{h}, \varepsilon}\left(\cdot, \varepsilon_{i}^{h}\right) \tilde{\Psi}_{i}^{h}-\chi_{i}\right\|_{L^{2} \cap L^{4}\left(\mathbb{R}^{3}\right)}=0 .
$$

Moreover, in this limit, the support of the test function $\varphi$ is definitely included in $\Omega^{h}$ and the scalar product at the l.h.s. can be written as

$$
\left(\left[-\Delta+U+\tilde{V}^{h}-\varepsilon_{i}^{h}\right] \varphi, 1_{\Omega^{h}} \tilde{\Psi}_{i}^{h}\right)_{L^{2}\left(\mathbb{R}^{3}\right)}=\left(\varphi,\left[-\Delta+U+\tilde{V}^{h}-\varepsilon_{i}^{h}\right] \tilde{\Psi}_{i}^{h}\right)_{L^{2}\left(\Omega^{h}\right)}=0
$$

where (1.10) has been taken into account. Previous relations and the elliptic regularity of the problem lead us to the following equation

$$
[-\Delta+U+G * \rho] \chi_{i}=\varepsilon_{S} \chi_{i} \quad \text { with } i=1, \ldots, N_{1}
$$

in $L^{2}\left(\mathbb{R}^{3}\right)$. The normalization and orthogonality properties of the eigenvectors $1_{\Omega^{h}} \tilde{\Psi}_{i \leq N_{1}}^{h}$ are preserved for $h \rightarrow 0$ and, in this limit, we have

$$
\begin{gathered}
\left\|\chi_{i}\right\|_{L^{2}\left(\mathbb{R}^{3}\right)}=1, \\
\left(\chi_{i}, \chi_{j}\right)_{L^{2}\left(\mathbb{R}^{3}\right)}=\delta_{i j} .
\end{gathered}
$$

The equations (3.15)-(3.16) define $\chi_{i}$ as an eigenvector related to the eigenvalue $\varepsilon_{S}$ of the limit Hamiltonian $H=-\Delta+U+G * \rho$. Let us focus our attention on the case $i=1$. Due to the characterization

$$
\tilde{\Psi}_{1}^{h} \geq 0 \quad \text { a.e. on } \Omega^{h}
$$


of the fundamental mode of $\tilde{H}^{h}$, and using the convergence of $1_{\Omega^{h}} \tilde{\Psi}_{1}^{h} \rightarrow \chi_{1}$ in $L^{2}\left(\mathbb{R}^{3}\right)$, it follows that: $\chi_{1} \geq 0$ a.e. in $\mathbb{R}^{3}$. Recalling that the unique non negative eigenvector coincides with the fundamental mode, we can identify $\chi_{1}$ with the fundamental mode of $H$ and $\varepsilon_{S}$ with its first eigenvalue. Then, the non degeneracy of the first energy level $\varepsilon_{S}$ and the linear independence of the vectors $\chi_{i}$ (see relation (3.17)) forces $N_{1}=1$. We conclude that the energy levels $\varepsilon_{i>1}^{h}$ definitely overcome the threshold $\varepsilon_{S}$ as $h \rightarrow 0$; therefore, the unique contribution to the charge density in the limit $h \rightarrow 0$, comes from the term: $A_{1}^{h}\left|\tilde{\Psi}_{1}^{h}\right|^{2}$ and, for a set $D$ verifying (3.2)-(3.1), the limit problem writes as (3.12).

\subsection{The limit equation}

The limit Schrödinger-Poisson equation obtained above essentially depends from the convergence of the charge density expressed by the conditions (3.1)-(3.2). The purpose, in what follows, is to analyze the uniqueness of this limit. In particular we will prove that any family of densities $\left(\rho^{h}\right)_{h \in D}$, fulfilling the convergence conditions (3.1)-(3.2), lead to the same limit equation (3.12) which will be shown to have a unique solution verifying (3.11). Our strategy consists in proving that the fundamental energy of the nonlinear limit Hamiltonian

$$
-\Delta+U+A G *|\chi|^{2}
$$

can be determined as an implicit function of the coupling parameter $A$. Then, making use of its monotonicity properties, we are able to prove that there exists a unique setting $\{A, \chi, W\}$ given by (3.11).

Let us denote with $H(W)$ the selfadjoint operator

$$
H(W)=-\Delta+U+W, \quad D(H(W))=H^{2}\left(\mathbb{R}^{3}\right) .
$$

We consider the functional $K_{a}: \dot{H}^{1}\left(\mathbb{R}^{3} ; \mathbb{R}\right) \rightarrow \mathbb{R}$

$$
\begin{gathered}
K_{a}(W)=\frac{1}{2} \int_{\mathbb{R}^{3}}(\nabla W)^{2} d x-a \varepsilon(W), \quad a \geq 0, \\
\varepsilon(W)=\inf \sigma(H(W)),
\end{gathered}
$$

where $\dot{H}^{1}\left(\mathbb{R}^{3} ; \mathbb{R}\right)$ is the homogeneous Sobolev space of real functions defined as the completion of $C_{0}^{\infty}\left(\mathbb{R}^{3}\right)$ with respect to the scalar norm

$$
\|W\|_{\dot{H}^{1}\left(\mathbb{R}^{3} ; \mathbb{R}\right)}=\left(\int_{\mathbb{R}^{3}}|\nabla W|^{2} d x\right)^{\frac{1}{2}} .
$$

This is an Hilbert space for the scalar product $(u, v)_{\dot{H}^{1}}=\int_{\mathbb{R}^{3}} \nabla u \cdot \nabla v d x$ and it is included in $L^{6}\left(\mathbb{R}^{3}\right)$. A simple Fourier decomposition $\hat{W}(\xi)=\hat{W}(\xi) 1_{|\xi| \leq 1}(\xi)+\hat{W}(\xi) 1_{|\xi|>1}(\xi)=\hat{W}_{1}(\xi)+\hat{W}_{2}(\xi)$ leads to

$$
\begin{aligned}
& W=W_{1}+W_{2}, \quad \hat{W}_{1} \in L^{1}\left(\mathbb{R}^{3}\right), \quad W_{2} \in L^{2}\left(\mathbb{R}^{3}\right), \\
& \text { with }\left\|\hat{W}_{1}\right\|_{L^{1}}+\left\|W_{2}\right\|_{L^{2}} \leq C\|W\|_{\dot{H}^{1}} .
\end{aligned}
$$

A direct application of Lemma B.1 implies

Lemma 3.4 The map $\varepsilon(W)$ defined by (3.20) belongs to $C^{0}\left(\dot{H}^{1}\left(\mathbb{R}^{3} ; \mathbb{R}\right),(-\infty, 0]\right)$ and is analytic in the open set

$$
S=\left\{W \in \dot{H}^{1}\left(\mathbb{R}^{3} ; \mathbb{R}\right), \varepsilon(W)<0\right\} .
$$


Proof. First note that the potential $U$, involved in the definition of $H(W)=-\Delta+U+W$ and $\varepsilon(W)=\inf \sigma(H(W))$, belongs to $\dot{H}^{1}(\mathbb{R})$. Hence Lemma B.1 can be used either with $H_{0}=-\Delta+U$ or $H_{0}=-\Delta$ while replacing $W$ with $W^{\prime}=U+W$. For $W \in \dot{H}^{1}\left(\mathbb{R}^{3}\right)$, the decomposition $(3.21)(3.22)$ allows to write for any $\psi \in H^{2}\left(\mathbb{R}^{3}\right)$

$$
\begin{aligned}
\left\|W_{1} \psi\right\|_{L^{2}} & \leq\left\|W_{1}\right\|_{L^{\infty}}\|\psi\|_{L^{2}} \leq C\|W\|_{\dot{H}^{1}}\|\psi\|_{L^{2}} \\
\left\|W_{2} \psi\right\|_{L^{2}} & \leq\left\|W_{2}\right\|_{L^{2}}\|\psi\|_{L^{\infty}} \leq\|W\|_{\dot{H}^{1}}\left[\delta\|\Delta \psi\|_{L^{2}}+C_{\delta}\|\psi\|_{L^{2}}\right]
\end{aligned}
$$

where the last inequality is a consequence of the interpolation inequality $\|\psi\|_{L^{\infty}} \leq C\|\Delta \psi\|_{L^{2}}^{a}\|\psi\|^{1-a}$ for $a=\frac{d}{4}<1$, in dimension $d \leq 3$. The function $W_{1}$ is bounded with $\lim _{x \rightarrow \infty} W_{1}(x)=0$ while $(1-\Delta)^{-1} W_{2}$ belongs to the space $\mathcal{L}^{2}\left(L^{2}\left(\mathbb{R}^{3}\right)\right)$ of Hilbert-Schmidt operator according to [18]. Hence $W=W_{1}+W_{2}$ is a relatively compact perturbation of $(-\Delta)$ in $\mathbb{R}^{3}$. All the conditions of Lemma B.1 are satisfied.

Corollary 3.5 Let $\psi(W)$ denote the first normalized eigenvector of the Hamiltonian $H(W)$. The $H^{2}\left(\mathbb{R}^{3}\right)$-valued map $W \mapsto \psi(W)$ is real analytic on $S$.

Proof. As in Lemma 3.4, the conditions of Lemma B.1 are satisfied. Then, the $L^{2}$-analyticity is a consequence of the Kato-Rellich theorem (see [17]-Theorem XII.8) applied there. The $H^{2}$ analyticity comes from the eigenvalue equation $-\Delta \psi(W)=\varepsilon(W) \psi(W)-(U+W) \psi(W)$.

We will use the previous result to investigate the minimization problem

$$
\inf _{W \in \dot{H}^{1}\left(\mathbb{R}^{3} ; \mathbb{R}\right)} K_{a}(W)
$$

with $K_{a}$ given by (3.19)-(3.20).

Proposition 3.6 The map (3.19)-(3.20) admits a unique global minimum in $\dot{H}^{1}\left(\mathbb{R}^{3} ; \mathbb{R}\right)$ noted $W_{a}$. In particular, if $W_{a} \in S$, it is the unique solution of the Schrödinger-Poisson problem

$$
\left\{\begin{array}{l}
-\Delta W_{a}=a\left|\psi_{a}\right|^{2} \\
H\left(W_{a}\right) \psi_{a}=\varepsilon_{a} \psi_{a}
\end{array} \quad \text { in } \mathbb{R}^{3}\right.
$$

with $\varepsilon_{a}=\varepsilon\left(W_{a}\right), \psi_{a}=\psi\left(W_{a}\right)$.

Proof. The map $K_{a}(W)$ can be explicitly written as

$$
K_{a}(W)=\frac{1}{2}\|W\|_{\dot{H}^{1}\left(\mathbb{R}^{3} ; \mathbb{R}\right)}^{2}-a \varepsilon(W), \quad a \geq 0 .
$$

It follows from Lemma 3.4 that $K_{a}(W)$ is continuous on $\dot{H}^{1}\left(\mathbb{R}^{3} ; \mathbb{R}\right)$. Moreover $-\varepsilon(W)$, defined as the supremum of affine maps, is convex. Therefore the sum: $K_{a}(W)=\frac{1}{2}\|W\|_{\dot{H}^{1}\left(\mathbb{R}^{3} ; \mathbb{R}\right)}^{2}-a \varepsilon(W)$ defines an $\alpha$-convex (with $\alpha=1$ ) continuous map on $\dot{H}^{1}\left(\mathbb{R}^{3} ; \mathbb{R}\right)$. Hence the minimization problem admits a unique solution $W_{a} \in \dot{H}^{1}\left(\mathbb{R}^{3} ; \mathbb{R}\right)$.

In particular, if the minimum $W_{a}$ is attained in the set $S$ - where $K_{a}(W)$ is differentiable - then (3.24) is equivalent to the Euler equation $d_{W} K_{a}\left(W_{a}\right)=0$ explicitly given by

$$
\int_{\mathbb{R}^{3}} \nabla W_{a} \cdot \nabla V-a d_{W} \varepsilon\left(W_{a}\right) \cdot V=0 \quad \forall V \in \dot{H}^{1}\left(\mathbb{R}^{3} ; \mathbb{R}\right),
$$

with $d_{W} \varepsilon\left(W_{a}\right) \cdot V$ denoting the action on $V$ of the differential map $d_{W} \varepsilon$ evaluated in $W_{a}$. This term can be expressed as

$$
d_{W} \varepsilon\left(W_{a}\right) \cdot V=\int_{\mathbb{R}^{3}}\left|\psi_{a}\right|^{2} V
$$

Equation (3.25) is a direct consequence of $(3.27),(3.28)$ and the density of $C_{0}^{\infty}\left(\mathbb{R}^{3}\right)$ in $\dot{H}^{1}\left(\mathbb{R}^{3} ; \mathbb{R}\right)$.

In what follows we consider the regularity properties, w.r.t. the variable $a$, of the maps $W_{a}, \varepsilon_{a}$ and $\psi_{a}$ introduced in the above Proposition. 
Lemma 3.7 Let $W_{a}$ denotes the minimum of the functional $K_{a}(W)$. With the notation of the Proposition 3.6, the maps $a \mapsto W_{a}$ and $a \mapsto \varepsilon_{a}$ are continuous in $\mathbb{R}^{+}$, and the maps $a \mapsto W_{a}$, $a \mapsto \psi_{a}$ and $a \mapsto \varepsilon_{a}$ are analytic in the domain $a \in \Sigma$

$$
\Sigma=\left\{a \in \mathbb{R}^{+} \mid \varepsilon_{a}<0\right\} .
$$

Proof. Let us consider the continuity problem at first. As already noticed in the proof of Proposition 3.6, $K_{a}$ is a continuous $\alpha$-convex map with $\alpha=1$. This implies

$$
K_{a}(W)-K_{a}\left(W_{a}\right) \geq \frac{1}{4}\left\|W-W_{a}\right\|_{\dot{H}^{1}\left(\mathbb{R}^{3} ; \mathbb{R}\right)}^{2}, \quad \forall W \in \dot{H}^{1}\left(\mathbb{R}^{3} ; \mathbb{R}\right) .
$$

Let us now fix $a_{0} \in \mathbb{R}^{+}$and consider the difference $W_{a}-W_{a_{0}}$ when $a$ belongs to a small neighbourhood of $a_{0}$. From the above estimate, we have

$$
\frac{1}{4}\left\|W_{a}-W_{a_{0}}\right\|_{\dot{H}^{1}\left(\mathbb{R}^{3} ; \mathbb{R}\right)}^{2} \leq K_{a_{0}}\left(W_{a}\right)-K_{a_{0}}\left(W_{a_{0}}\right) \leq\left|K_{a}\left(W_{a}\right)-K_{a_{0}}\left(W_{a_{0}}\right)\right|+\left|a_{0}-a\right|\left|\varepsilon_{a}\right| .
$$

Here we notice that for $W=0$, the value $K_{a}(0)$ is defined by the first eigenvalue of the Hamiltonian $-\Delta+U$ and belongs to the bounded interval $\left(0, a\|U\|_{L^{\infty}}\right]$. Therefore, for any $a \in \mathbb{R}^{+}$, the infimum value of the map $K_{a}$ is bounded by $a\|U\|_{L^{\infty}}$. This circumstance implies

$$
a\left|\varepsilon_{a}\right| \leq K_{a}\left(W_{a}\right) \leq a\|U\|_{L^{\infty}} .
$$

The map $a \mapsto K_{a}\left(W_{a}\right)$ is non negative and concave as the infimum of non negative affine maps, $K_{a}\left(W_{a}\right)=\inf _{W \in \dot{H}^{1}\left(\mathbb{R}^{3}, \mathbb{R}\right)}-\varepsilon(W) a+\frac{1}{2}\|W\|_{\dot{H}^{1}}^{2}$. Therefore, it is continuous and previous relations lead us to

$$
\lim _{a \rightarrow a_{0}}\left\|W_{a}-W_{a_{0}}\right\|_{\dot{H}^{1}\left(\mathbb{R}^{3} ; \mathbb{R}\right)}^{2}=0 .
$$

The continuity of the map $a \mapsto \varepsilon_{a}$ is a direct consequence of this result and the continuity of $\varepsilon(W)$ (Lemma 3.4).

Next we investigate the analyticity problem. Owing to the continuity of $\varepsilon_{a}, \Sigma$ is an open and non empty set, with $0 \in \Sigma$ as a consequence of the condition (1.16). Let $a \in \Sigma$; as remarked in the proof of Proposition 3.6, the corresponding minimum $W_{a} \in S$ satisfies the Euler equation

$$
d_{W} K_{a}\left(W_{a}\right)=0 .
$$

Let $I_{a}$ and $I_{W_{a}}$ denote two open neighbourhoods of $a$ and $W_{a}$ in $\Sigma$ and $S$ respectively. For a differentiable function $f$ from $\dot{H}^{1}\left(\mathbb{R}^{3} ; \mathbb{R}\right)$ to $\mathbb{R}$ and for $W \in \dot{H}^{1}\left(\mathbb{R}^{3} ; \mathbb{R}\right), \nabla_{\dot{H}^{1}} f(W) \in \dot{H}^{1}\left(\mathbb{R}^{3} ; \mathbb{R}\right)$ denotes the gradient of $f$ which represents the scalar product on $\dot{H}^{1}\left(\mathbb{R}^{3} ; \mathbb{R}\right)$ of the differential $d f(W): \dot{H}^{1}\left(\mathbb{R}^{3} ; \mathbb{R}\right) \rightarrow \mathbb{R}$. Due to Lemma 3.4 , the map $K_{\alpha}: I_{a} \rightarrow I_{W_{a}}$ is analytic and $\nabla_{\dot{H}^{1}} K_{\alpha}(W)=$ $W-\alpha \nabla_{\dot{H}^{1}} \varepsilon(W)$ is well defined and analytic on $I_{W_{a}}$. We introduce the map $F: I_{a} \times I_{W_{a}} \rightarrow$ $\dot{H}^{1}\left(\mathbb{R}^{3} ; \mathbb{R}\right)$

From (3.34), we have

$$
F(\alpha, W)=W-\alpha \nabla_{\dot{H}^{1}} \varepsilon(W)
$$

$$
F\left(a, W_{a}\right)=0
$$

moreover

$$
d_{W} F\left(a, W_{a}\right)=I-a \nabla_{\dot{H}^{1}}^{2} \varepsilon\left(W_{a}\right) \in \mathcal{L}\left(\dot{H}^{1}, \dot{H}^{1}\right) .
$$

The convexity of $-\varepsilon(W)$ implies

$$
-\left(\nabla_{\dot{H}^{1}}^{2} \varepsilon\left(W_{a}\right) V, V\right)_{\dot{H}^{1}} \geq 0
$$

This ensures the coercivity of the continuous symmetric bilinear form $\left(V, V^{\prime}\right) \mapsto\left(d_{W} F\left(a, W_{a}\right) V, V^{\prime}\right)_{\dot{H}^{1}}$. Therefore, from Lax-Milgram Theorem, we know that $d_{W} F\left(a, W_{a}\right)$ is invertible and an implicit function technique (e.g. in [15]) can be implemented to state that there exists an open neighbourhood of $a, U_{a}$, and an analytic map $\alpha \mapsto W_{\alpha}$ from $U_{a}$ to $I_{W_{a}}$ such that

$$
F\left(\alpha, W_{\alpha}\right)=0, \quad \forall \alpha \in U_{a}
$$


The function $W_{\alpha} \in \dot{H}^{1}\left(\mathbb{R}^{3} ; \mathbb{R}\right)$ solves the Euler equation (3.34) and, by Proposition 3.6, is the unique minimum of $K_{\alpha}$; moreover it is real analytic w.r.t. $\alpha \in U_{a}$. The analyticity of the maps $a \mapsto \varepsilon_{a}$ and $a \mapsto \psi_{a}$ easily follows from this result and those obtained in Lemma 3.4 and Corollary 3.5 .

Lemma 3.8 The analytic function $\varepsilon_{a}, a \in \Sigma$, defined in Lemma 3.7, is strictly increasing.

Proof. We recall that $\varepsilon_{a}$ is defined as the composite map $\varepsilon\left(W_{a}\right)$ where $W_{a}$ is the minimum of the functional $K_{a}(W)$. We can apply the rule of derivation of composite maps (e.g. in [1]) and the relation (3.28) to write

$$
\varepsilon_{a}^{\prime}=d_{W} \varepsilon\left(W_{a}\right) \cdot W_{a}^{\prime}=\int_{\mathbb{R}^{3}}\left|\psi_{a}\right|^{2} W_{a}^{\prime},
$$

$W_{a}^{\prime}$ denoting the variation of $W_{a}$. Differentiating the minimum condition (3.27) w.r.t. $a$ and using (3.28) we obtain the equation

$$
\int_{\mathbb{R}^{3}} \nabla W_{a}^{\prime} \cdot \nabla V-\int_{\mathbb{R}^{3}}\left|\psi_{a}\right|^{2} V-a \frac{d}{d a} \int_{\mathbb{R}^{3}}\left|\psi_{a}\right|^{2} V=0 \quad \forall V \in \dot{H}^{1}\left(\mathbb{R}^{3}\right),
$$

where the last term at the l.h.s. can be rewritten as

$$
\frac{d}{d a} \int_{\mathbb{R}^{3}}\left|\psi_{a}\right|^{2} V=\frac{d}{d a} d_{W} \varepsilon\left(W_{a}\right) \cdot V=d_{W}^{2} \varepsilon\left(W_{a}\right) \cdot\left(V, W_{a}^{\prime}\right) .
$$

Setting $V=W_{a}^{\prime}$ in (3.36) and using the relations (3.35)-(3.37), we obtain the following representation of $\varepsilon_{a}^{\prime}$

$$
\varepsilon_{a}^{\prime}=\int_{\mathbb{R}^{3}} \nabla W_{a}^{\prime} \cdot \nabla W_{a}^{\prime}-a d_{W}^{2} \varepsilon\left(W_{a}\right) \cdot\left(W_{a}^{\prime}, W_{a}^{\prime}\right) .
$$

The r.h.s. of this expression defines the second differential of $K_{a}(W)$ evaluated in the point $W_{a}$ and acting on the couple $\left(W_{a}^{\prime}, W_{a}^{\prime}\right)$. From the convexity of this map, we know that

$$
d_{W}^{2} K_{a}\left(W_{a}\right) \cdot(V, V) \geq 0 \quad \forall V \in \dot{H}^{1}\left(\mathbb{R}^{3} ; \mathbb{R}\right)
$$

and the condition $\varepsilon_{a}^{\prime} \geq 0$ follows. This condition, together with the analyticity of $\varepsilon_{a}$ lead us to the statement of the Lemma.

We conclude this Section giving the proof of Theorem 1.1.

Proof of Theorem 1.1. We start considering the second statement of the Theorem. Making use of the notations introduced above, the solution to (1.17) identifies with a couple $\left(A, W_{A}\right)$ fulfilling the minimization problem

$$
\inf _{W \in \dot{H}^{1}\left(\mathbb{R}^{3} ; \mathbb{R}\right)} K_{A}(W),
$$

and the condition

$$
\varepsilon_{A}=\varepsilon_{S}
$$

where $\varepsilon_{S}$ is the fixed datum. In Proposition 3.3, it has been shown that there exists at least one setting $\left(A, G * A|\chi|^{2}\right)$ - where $A$ and $\chi$ are defined as a limit in $(3.11)$ - solving this problem. Due to the Lemmas 3.7 and 3.8, the function $a \longmapsto \varepsilon_{a}$ is continuous on $\mathbb{R}^{+}$, strictly increasing on the subset $\Sigma \subset \mathbb{R}^{+}$and null outside $\Sigma$ (since $W_{a}$ is a $H_{0}$-relatively compact)

$$
\left.\varepsilon_{a}\right|_{\mathbb{R}^{+} \backslash \Sigma}=0
$$

Moreover, from the definition (1.14) and the assumption (1.16), it is known that: $\varepsilon_{0}=e_{1}<\varepsilon_{S}$. The previous characterization of $\varepsilon_{a}$ forces the solution, $A$, to the equation

$$
\varepsilon_{A}=\varepsilon_{S}
$$

to be unique and strictly positive. Then, the uniqueness of the couple $\left(A, W_{A}\right)$ follows from the uniqueness of the minimum of $K_{A}(W)$. 
The third statement relies on an ad absurdum argument. If $1_{\Omega^{h}} \tilde{V}^{h}$ does not converge to the potential $W$ determined by the unique solution to (1.17), then we can find a set $S \subset\left(0, h_{0}\right], 0 \in \bar{S}$, such that

$$
\left\|1_{\Omega^{h}} \tilde{V}^{h}-W\right\|_{L^{\infty}\left(\mathbb{R}^{3}\right)}>c, \quad \forall h \in S .
$$

for some positive constant $c$. On the other hand, it is possible to extract a subset $D \subset S, 0 \in \bar{D}$, such that (3.1) and (3.2) are verified. According to Proposition 3.1, Proposition 3.3 and the previous uniqueness result, the following limit holds

$$
\lim _{\substack{h \rightarrow 0 \\ h \in D}}\left\|1_{\Omega^{h}} \tilde{V}^{h}-W\right\|_{L^{\infty}\left(\mathbb{R}^{3}\right)}=0
$$

which contradicts the initial assumption.

The first statement of the Theorem is a consequence of the third one and Corollary 3.2. Finally, the fourth statement is an application of Proposition 3.3, once the third statement holds true.

\section{The asymptotic problem in the 2-D case}

The analysis of the asymptotic behaviour of the Poisson potential in the 2-D case needs a different approach w.r.t. the one followed in the previous Section. This is essentially due to the fact that the singularity of the integral kernel of $(-\Delta)^{-1}$, in dimension 2 , prevents us to use the estimate (3.3) and a global Fourier analysis approach in the study of the problem. Let consider the rescaled density $r^{h}$

$$
r^{h}=\sum_{i \leq N_{0}} B_{i}^{h}\left|\mathbb{I}_{\Omega^{h}, \varepsilon_{S}}\left(\cdot, \varepsilon_{i}^{h}\right) \tilde{\Psi}_{i}^{h}\right|^{2},
$$

where, due to the results of Lemma 2.7, the coefficients

$$
B_{i}^{h}=|\ln h| A_{i}^{h}
$$

are uniformly bounded w.r.t. $h \in\left(0, h_{0}\right]$. It is possible to extract out of any $S \subset\left(0, h_{0}\right], 0 \in \bar{S}$, a subset $D \subset S, 0 \in \bar{D}$, such that

$$
\lim _{\substack{h \rightarrow 0 \\ h \in D}} B_{i}^{h}=B_{i} .
$$

It is worthwhile to stress that the convergence conditions (4.3) contain the existence of a uniform bound for $B_{i}^{h}$ and $B_{i}$ w.r.t. $h$

$$
B_{i}^{h}, B_{i} \leq C, \quad \forall h \in D
$$

This property, together with the normalization condition $\left\|\tilde{\Psi}_{i}^{h}\right\|_{L^{2}\left(\Omega^{h}\right)}=1$, will be extensively used in what follows. As already noticed (see the proof of Lemma 2.9$)$, the family $\left(\mathbb{I}_{\Omega^{h}, \varepsilon}\left(\cdot, \varepsilon_{i}^{h}\right) \tilde{\Psi}_{i}^{h}\right)_{h \in\left(0, h_{0}\right]}$ is bounded in $L^{2} \cap L^{4}\left(\mathbb{R}^{2}\right)$ uniformly w.r.t. the parameter $h$. Then, due to the definition (4.1), the rescaled density $\left(r^{h}\right)_{h \in\left(0, h_{0}\right]}$ is bounded in $L^{1} \cap L^{2}\left(\mathbb{R}^{2}\right)$. Moreover, it follows from relation

$$
1_{\Omega^{h}} \rho^{h}=\left(\ln \frac{1}{h}\right)^{-1} r^{h},
$$

that

$$
\left\|1_{\Omega^{h}} \rho^{h}\right\|_{L^{1} \cap L^{2}\left(\mathbb{R}^{2}\right)}=\mathcal{O}\left(|\ln h|^{-1}\right)
$$

as $h \rightarrow 0$. The above result proves that, unlike the $3-\mathrm{D}$ case, if any non null limit potential exists, it will not satisfy a Poisson problem.

In this analysis, we consider the Poisson potential $\tilde{V}^{h}$ defined, in terms of the rescaled density, by the equation

$$
\left\{\begin{array}{l}
-\Delta \tilde{V}^{h}=\left(\ln \frac{1}{h}\right)^{-1} r^{h} \quad \text { in } \Omega^{h} \\
\left.\tilde{V}^{h}\right|_{\partial \Omega^{h}}=0 .
\end{array}\right.
$$


Our strategy consists into a direct estimate of the $L^{\infty}$-norm of the contribution to $\tilde{V}^{h}$ given by the source term in a region of size $\mathcal{O}\left(\ln \frac{1}{h}\right)$. The size $\mathcal{O}\left(\ln \frac{1}{h}\right)$ appears naturally since it is small enough to apply the multipole expansion of the $(-\Delta)^{-1}$-Green kernel on $\partial \Omega^{h}$, and big enough to exploit the exponential decay of the eigenfunctions outside a region of this size. In particular, exploiting the compensation between the logaritmic singularity of the Laplacian's Green function and the scaling factor $\left(\ln \frac{1}{h}\right)^{-1}$ appearing in (4.6), we are able to obtain an explicit asymptotic picture of the Poisson potential, as $h \rightarrow 0$, both at the classical and the quantum scales.

In what follows $B_{r}$ and $B(x, r)$ denote the balls of radius $r$ centered, respectively, in the origin and in the point $x$ of $\mathbb{R}^{2}$. Moreover, we fix $R$ and $R^{\prime}$ such that the following inclusions hold

$$
B_{\frac{R}{h}} \subset \Omega^{h} \subset B_{\frac{R^{\prime}}{h}} .
$$

Finally, setting $R^{h}=\kappa\left(\ln \frac{1}{h}\right)$ for some $\kappa>0$, the inclusions

$$
B_{R^{h}} \subset B_{\frac{R}{h}} \subset \Omega^{h}
$$

hold for all $h \in\left(0, h_{0}\right]$ when $h_{0}$ is chosen small enough, depending on $\kappa$ and $R$. Next, we consider the decomposition

$$
\phi_{1}^{h}=r^{h} I_{B_{R^{h}}}, \quad \phi_{2}^{h}=r^{h}\left(1-I_{B_{R^{h}}}\right),
$$

where $I_{B_{R^{h}}}$ is the characteristic function of the ball $B_{R^{h}}$ and $r^{h}$ is given by (4.1). The Poisson potential $\tilde{V}^{h}$ can be written as

$$
\tilde{V}^{h}=W_{1}^{h}+W_{2}^{h}
$$

with

$$
\left\{\begin{array}{l}
-\Delta W_{j}^{h}=\left(\ln \frac{1}{h}\right)^{-1} \phi_{j}^{h} \quad \text { in } \Omega^{h}, \\
\left.W_{j}^{h}\right|_{\partial \Omega^{h}}=0 .
\end{array}\right.
$$

The next Lemmas give an asymptotic characterization as $h \rightarrow 0$ of the fields $W_{j=1,2}^{h}$ and $W^{h}$.

Lemma 4.1 For any $\kappa>0$, the function $W_{2}^{h}$, defined by (4.1), (4.9) and (4.11) tends uniformly to 0 on $\Omega^{h}$ as $h \rightarrow 0$ with the following asymptotic order

$$
\left\|W_{2}^{h}\right\|_{L^{\infty}\left(\Omega^{h}\right)}=\mathcal{O}\left(h^{\gamma}\right)
$$

for some constant $\gamma \in(0,1)$.

Proof. Let us introduce the auxiliary function

$$
\tilde{W}_{2}^{h}=\left(\ln \frac{1}{h}\right)^{-1} G * \phi_{2}^{h},
$$

where $G$ denotes the Green kernel of the Laplacian in $\mathbb{R}^{2}: G(x, y)=-\frac{1}{2 \pi} \ln |x-y|$. The difference $W_{2}^{h}-\tilde{W}_{2}^{h}$ satisfies the equation

$$
\left\{\begin{array}{l}
-\Delta\left(W_{2}^{h}-\tilde{W}_{2}^{h}\right)=0 \quad \text { in } \Omega^{h} \\
W_{2}^{h}-\left.\tilde{W}_{2}^{h}\right|_{\partial \Omega^{h}}=-\left.\tilde{W}_{2}^{h}\right|_{\partial \Omega^{h}},
\end{array}\right.
$$

and, due to the maximum principle, we get

$$
\left\|W_{2}^{h}\right\|_{L^{\infty}\left(\Omega^{h}\right)} \leq\left\|W_{2}^{h}-\tilde{W}_{2}^{h}\right\|_{L^{\infty}\left(\Omega^{h}\right)}+\left\|\tilde{W}_{2}^{h}\right\|_{L^{\infty}\left(\Omega^{h}\right)} \leq\left\|\tilde{W}_{2}^{h}\right\|_{L^{\infty}\left(\partial \Omega^{h}\right)}+\left\|\tilde{W}_{2}^{h}\right\|_{L^{\infty}\left(\Omega^{h}\right)} .
$$

For $R^{\prime}$ compliant with condition (4.7), previous inequality gives

$$
\left\|W_{2}^{h}\right\|_{L^{\infty}\left(\Omega^{h}\right)} \leq 2\left\|\tilde{W}_{2}^{h}\right\|_{L^{\infty}\left(B_{\frac{R^{\prime}}{h}}\right)} .
$$


In the next estimate the values of $\tilde{W}_{2}^{h}$ over $B_{\frac{R^{\prime}}{h}}$ are considered. Let $x \in B_{\frac{R^{\prime}}{h}}$; according to the explicit definitions of $\tilde{W}_{2}^{h}, \phi_{2}^{h}$ and $r^{h}$, the uniform boundedness of the coefficients $B_{i \leq N_{0}}^{h}$ and the decay estimate (A.18), we have

$$
\begin{aligned}
& \left|\tilde{W}_{2}^{h}(x)\right|=\left|\frac{|\ln h|^{-1}}{2 \pi} \int_{\mathbb{R}^{2}} \ln \right| x-y\left|\phi_{2}^{h}(y) d y\right| \leq \frac{|\ln h|^{-1}}{2 \pi} \sum_{\substack{i \leq N_{0} \\
\varepsilon_{i}^{h}<\varepsilon_{S}}} B_{i}^{h} \int_{\mathbb{R}^{2} \backslash B_{R^{h}}}|\ln | x-y||\left|1_{\Omega^{h}} \tilde{\Psi}_{i}^{h}(y)\right|^{2} d y \\
& \leq \frac{|\ln h|^{-1}}{2 \pi} \sum_{\substack{i \leq N_{0} \\
\varepsilon_{i}^{h}<\varepsilon_{S}}} B_{i}^{h} e^{-2 c_{0} R^{h}} \int_{\mathbb{R}^{2} \backslash B_{R^{h}}}|\ln | x-y||\left|1_{\Omega^{h}} e^{c_{0}|y|} \tilde{\Psi}_{i}^{h}(y)\right|^{2} d y \\
& \leq C \frac{e^{-2 c_{0} R^{h}}}{|\ln h|} \sum_{\substack{i \leq N_{0} \\
\varepsilon_{i}^{h}<\varepsilon_{S}}}\left[\|\ln |x-y|\|_{L^{2}(B(x, 1))}\left\|e^{c_{0}|x|} \tilde{\Psi}_{i}^{h}\right\|_{L^{4}\left(\Omega^{h}\right)}^{2}\right. \\
& \left.+\int_{\mathbb{R}^{2} \backslash\left(B(x, 1) \cup B_{R^{h}}\right)}|\ln | x-y||\left|1_{\Omega^{h}} e^{c_{0}|y|} \tilde{\Psi}_{i}^{h}(y)\right|^{2} d y\right] \\
& \leq C \frac{e^{-2 c_{0} R^{h}}}{|\ln h|} \sum_{\substack{i \leq N_{0} \\
\varepsilon_{i}^{h}<\varepsilon_{S}}}\left[1+\ln \frac{2 R^{\prime}}{h} \int_{\mathbb{R}^{2} \backslash\left(B(x, 1) \cup B_{R^{h}}\right)}\left|1_{\Omega^{h}} e^{c_{0}|y|} \tilde{\Psi}_{i}^{h}(y)\right|^{2} d y\right] \\
& \leq C \frac{e^{-2 c_{0} R^{h}}}{|\ln h|} \sum_{\substack{i \leq N_{0} \\
\varepsilon_{i}^{h}<\varepsilon_{S}}}\left[1+\ln \frac{2 R^{\prime}}{h}\left\|e^{c_{0}|x|} \tilde{\Psi}_{i}^{h}\right\|_{L^{2}\left(\Omega^{h}\right)}^{2}\right] \\
& \leq C e^{-2 c_{0} R^{h}}\left(|\ln h|^{-1}+1\right),
\end{aligned}
$$

where at each step $C$ is a suitable positive constant. Combining this estimate with (4.14), it easily follows that

$$
\left\|W_{2}^{h}\right\|_{L^{\infty}\left(\Omega^{h}\right)}=\mathcal{O}\left(e^{-c_{0} \kappa|\ln h|}\right)
$$

as $h \rightarrow 0$.

Let us introduce the function

$$
\tilde{W}_{1}^{h}=\left(\ln \frac{1}{h}\right)^{-1} G * \phi_{1}^{h}
$$

and the constant

$$
\theta=\frac{1}{2 \pi} \sum_{i \leq N_{0}} B_{i}
$$

where, $G$ denotes the Green kernel of the Laplacian in $\mathbb{R}^{2}: G(x, y)=-\frac{1}{2 \pi} \ln |x-y|$, while $B_{i}$ are the limits (4.3) of the coefficients $B_{i}^{h}$.

Lemma 4.2 For any $\kappa>0$, let $W_{1}^{h}$ and $\tilde{W}_{1}^{h}$ be defined by (4.1), (4.9), (4.11) and (4.15), and assume $R, R^{\prime}$ and $h_{0}$ satisfy the conditions (4.7) and (4.8). For a set $D$ such that the condition (4.3) is verified, the following limits hold

$$
\begin{gathered}
\lim _{\substack{h \rightarrow 0 \\
h \in D}}\left\|\tilde{W}_{1}^{h}+\theta\right\|_{L^{\infty}\left(B_{\left.\frac{R^{\prime}}{h} \backslash B_{\frac{R}{h}}\right)}\right.}=0, \\
\lim _{\substack{h \rightarrow 0 \\
h \in D}}\left\|W_{1}^{h}-\left(\tilde{W}_{1}^{h}+\theta\right)\right\|_{L^{\infty}\left(\Omega^{h}\right)}=0, \\
\lim _{\substack{h \rightarrow 0 \\
h \in D}}\left\|\tilde{W}_{1}^{h}\right\|_{L^{\infty}\left(B_{R^{h}}\right)}=0 .
\end{gathered}
$$


Proof. Take the function $\tilde{W}_{1}^{h}$

$$
\tilde{W}_{1}^{h}(x)=-\frac{\left(\ln \frac{1}{h}\right)^{-1}}{2 \pi} \sum_{\substack{i \leq N_{0} \\ \varepsilon_{i}^{h}<\varepsilon_{S}}} B_{i}^{h} \int_{B_{R^{h}}} \ln |x-y|\left|\tilde{\Psi}_{i}^{h}(y)\right|^{2} d y
$$

on the set $B_{\frac{R^{\prime}}{h}} \backslash B_{\frac{R}{h}}$. Let $(r, \vartheta)$ and $\left(r^{\prime}, \vartheta^{\prime}\right)$ denote the polar coordinates of the points $x$ and $y$ respectively. For $x \in B_{\frac{R^{\prime}}{h}} \backslash B_{\frac{R}{h}}$ and $y \in B_{R^{h}}$, the ratio $\frac{r^{\prime}}{r}$ is estimated by: $\frac{r^{\prime}}{r} \leq \frac{\kappa}{R} h\left(\ln \frac{1}{h}\right)$, and the multipole expansion

$$
\ln |x-y|=\ln r-\sum_{k=1}^{+\infty} \frac{1}{k}\left(\frac{r^{\prime}}{r}\right)^{k}\left[\cos k \vartheta \cos k \vartheta^{\prime}+\sin k \vartheta \sin k \vartheta^{\prime}\right]
$$

is uniformly convergent for $\frac{\kappa}{R} h\left(\ln \frac{1}{h}\right)<1$. According to this expansion, the values of $\tilde{W}_{1}^{h}$ over the set $B_{\frac{R^{\prime}}{h}} \backslash B_{\frac{R}{h}}$ can be expressed as follows

$$
\left.\tilde{W}_{1}^{h}(x)\right|_{x \in B_{\frac{R^{\prime}}{h} \backslash B_{\frac{R}{h}}}}=-\frac{\left(\ln \frac{1}{h}\right)^{-1}}{2 \pi} \sum_{\substack{i \leq N_{0} \\ \varepsilon_{i}^{h}<\varepsilon_{S}}} B_{i}^{h} \ln |x| \int_{B_{R^{h}}}\left|\tilde{\Psi}_{i}^{h}(y)\right|^{2} d y+o\left(|\ln h|^{-1}\right) .
$$

Replace $\ln |x|$ with

$$
\ln |x|=\left(\ln \frac{1}{h}\right)\left(1+\left(\ln \frac{1}{h}\right)^{-1} \ln h|x|\right) .
$$

When $x$ belongs to a domain of size $\sim \frac{1}{h}$, the quantity $\ln h|x|$ is uniformly bounded as $h \rightarrow 0$. Therefore we get

$$
\left.\tilde{W}_{1}^{h}(x)\right|_{x \in B_{\frac{R^{\prime}}{h} \backslash B_{\frac{R}{h}}}}=-\frac{1}{2 \pi} \sum_{\substack{i \leq N_{0} \\ \varepsilon_{i}^{h}<\varepsilon_{S}}} B_{i}^{h} \int_{B_{R^{h}}}\left|\tilde{\Psi}_{i}^{h}(y)\right|^{2} d y+o\left(|\ln h|^{-1}\right) .
$$

Making use of this expression, the norm $\left\|\tilde{W}_{1}^{h}+\theta\right\|_{L^{\infty}\left(B_{\left.\frac{R^{\prime}}{h} \backslash B_{\frac{R}{h}}\right)}\right.}$ admits the estimate

$$
\begin{aligned}
\left\|\tilde{W}_{1}^{h}+\theta\right\|_{L^{\infty}\left(B_{\left.\frac{R^{h}}{h} \backslash B_{\frac{R}{h}}\right)}\right.} & \leq\left.\left|\frac{1}{2 \pi} \sum_{\substack{i \leq N_{0} \\
\varepsilon_{i}^{h}<\varepsilon_{S}}} B_{i}^{h} \int_{B_{R^{h}}}\right| \tilde{\Psi}_{i}^{h}(y)\right|^{2} d y-\theta \mid+o\left(|\ln h|^{-1}\right) \\
& \leq \frac{1}{2 \pi} \sum_{\substack{i \leq N_{0} \\
\varepsilon_{i}^{h}<\varepsilon_{S}}}\left|B_{i}^{h}\left\|\tilde{\Psi}_{i}^{h}\right\|_{L^{2}\left(B_{R^{h}}\right)}^{2}-B_{i}\right|+o\left(|\ln h|^{-1}\right) .
\end{aligned}
$$

Taking into account the exponential decay of the eigenfunctions $\tilde{\Psi}_{i}^{h}$ with $\varepsilon_{i}^{h}<\varepsilon_{S}$ and the limit conditions $B_{i}^{h} \underset{h \rightarrow 0, h \in D}{\longrightarrow} B_{i}$, we have

$$
\begin{aligned}
&\left|B_{i}^{h}\left\|\tilde{\Psi}_{i}^{h}\right\|_{L^{2}\left(B_{R^{h}}\right)}^{2}-B_{i}\right| \leq B_{i}^{h}\left|\left\|\tilde{\Psi}_{i}^{h}\right\|_{L^{2}\left(B_{R^{h}}\right)}^{2}-1\right|+\left|B_{i}^{h}-B_{i}\right| \\
&=B_{i}^{h}\left|\left\|\tilde{\Psi}_{i}^{h}\right\|_{L^{2}\left(B_{R^{h}}\right)}^{2}-\left\|\tilde{\Psi}_{i}^{h}\right\|_{L^{2}\left(\mathbb{R}^{2}\right)}^{2}\right|+\left|B_{i}^{h}-B_{i}\right|=B_{i}^{h}\left\|\tilde{\Psi}_{i}^{h}\right\|_{L^{2}\left(\mathbb{R}^{2} \backslash B_{R^{h}}\right)}^{2}+\left|B_{i}^{h}-B_{i}\right| \\
& \leq B_{i}^{h} e^{-2 c_{0} R^{h}}\left\|e^{c_{0}|\cdot|} \tilde{\Psi}_{i}^{h}\right\|_{L^{2}\left(\mathbb{R}^{2}\right)}^{2}+\left|B_{i}^{h}-B_{i}\right| .
\end{aligned}
$$


These inequalities lead us to (4.17).

Let us consider (4.18). The function $W_{1}^{h}-\left(\tilde{W}_{1}^{h}+\theta\right)$ is the unique solution to the problem

$$
\left\{\begin{array}{l}
-\Delta u=0 \quad \text { in } \Omega^{h} \\
\left.u\right|_{\partial \Omega^{h}}=-\left.\left(\tilde{W}_{1}^{h}+\theta\right)\right|_{\partial \Omega^{h}}
\end{array}\right.
$$

Using the Maximum Principle and (4.17), we get

$$
\lim _{\substack{h \rightarrow 0 \\ h \in D}}\left\|W_{1}^{h}-\left(\tilde{W}_{1}^{h}+\theta\right)\right\|_{L^{\infty}\left(\Omega^{h}\right)} \leq \lim _{\substack{h \rightarrow 0 \\ h \in D}}\left\|\tilde{W}_{1}^{h}+\theta\right\|_{L^{\infty}\left(\partial \Omega^{h}\right)} \leq \lim _{\substack{h \rightarrow 0 \\ h \in D}}\left\|\tilde{W}_{1}^{h}+\theta\right\|_{L^{\infty}\left(B_{\left.\frac{R^{\prime}}{h} \backslash B_{\frac{R}{h}}\right)}\right.}=0 .
$$

Concerning the last limit, (4.19), we notice that, for any $x \in B_{R^{h}}$, the following estimate holds

$$
\begin{aligned}
\left|\tilde{W}_{1}^{h}(x)\right| \leq \frac{\left(\ln \frac{1}{h}\right)^{-1}}{2 \pi} \sum_{\substack{i \leq N_{0} \\
\varepsilon_{i}^{h}<\varepsilon_{S}}} B_{i}^{h} \int_{B_{R^{h}}}|\ln | x-y||\left|\tilde{\Psi}_{i}^{h}(y)\right|^{2} d y \\
\leq C\left(\ln \frac{1}{h}\right)^{-1} \sum_{\substack{i \leq N_{0} \\
\varepsilon_{i}^{h}<\varepsilon_{S}}}\left(\|\ln |x-y|\|_{L^{2}(B(x, 1))}\left\|\tilde{\Psi}_{i}^{h}\right\|_{L^{4}\left(\Omega^{h}\right)}^{2}\right. \\
\leq C\left(\ln \frac{1}{h}\right)^{-1} \sum_{\substack{i \leq N_{0} \\
\varepsilon_{i}^{h}<\varepsilon_{S}}}\left(1+\ln \left(2 R^{h}\right) \int_{B_{R^{h}} \backslash B(x, 1)}\left|\tilde{\Psi}_{i}^{h}(y)\right|^{2} d y\right) \\
\leq C\left(\ln \frac{1}{h}\right)^{-1} \sum_{\substack{i \leq N_{0} \\
\varepsilon_{i}^{h}<\varepsilon_{S}}}\left(1+\ln \left(2 R^{h}\right)\left\|\tilde{\Psi}_{i}^{h}\right\|_{L^{2}\left(\Omega^{h}\right)}^{2}\right)
\end{aligned}
$$

$C$ denoting, at each step, a suitable positive constant. The relevant term at the r.h.s. of this expression is the product: $\left(\ln \frac{1}{h}\right)^{-1} \ln \left(2 R^{h}\right)$, which, due to the definition of $R^{h}$, is proportional to an infinitesimal of type $\frac{1}{x} \ln x$ with $x=2 \kappa\left(\ln \frac{1}{h}\right)$ as $h \rightarrow 0$. This concludes the proof.

The results of previous Lemmas will help us to characterize the asymptotic shape of the Poisson potential $\tilde{V}^{h}$ as $h \rightarrow 0$.

Proposition 4.3 For a set $D$ such that the condition (4.3) is verified with $h_{0}>0$ small enough, the family of potentials $\left(1_{\Omega^{h}} \tilde{V}^{h}\right)_{h \in D}$ is bounded in $\mathbb{R}^{2}$ and uniformly convergent on any compact domain to the constant potential $\theta$ defined by (4.16)). More precisely, for any $\kappa>0$, setting $R^{h}=\kappa\left(\ln \frac{1}{h}\right)$, the family $\left(\tilde{V}^{h}\right)_{h \in D}$ satisfies the limit

$$
\lim _{\substack{h \rightarrow 0 \\ h \in D}}\left\|\tilde{V}^{h}-\theta\right\|_{L^{\infty}\left(B_{R^{h}}\right)}=0
$$

Proof. Set $\kappa>0, R^{h}=\kappa\left(\ln \frac{1}{h}\right)$. Let $R$ and $h_{0}$ satisfy the condition (4.8). The decomposition (4.10) and the results of the Lemmas 4.1 and 4.2, give the following auxiliary estimates

- Consider the difference $\tilde{V}^{h}-\theta$ on $B_{R^{h}}$

$$
\begin{aligned}
\left\|\tilde{V}^{h}-\theta\right\|_{L^{\infty}\left(B_{R^{h}}\right)} & \leq\left\|W_{1}^{h}-\theta\right\|_{L^{\infty}\left(B_{R^{h}}\right)}+\left\|W_{2}^{h}\right\|_{L^{\infty}\left(B_{R^{h}}\right)} \\
& \leq\left\|W_{1}^{h}-\theta\right\|_{L^{\infty}\left(B_{R^{h}}\right)}+\left\|W_{2}^{h}\right\|_{L^{\infty}\left(\Omega^{h}\right)}=\left\|W_{1}^{h}-\theta\right\|_{L^{\infty}\left(B_{R^{h}}\right)}+\mathcal{O}\left(h^{\gamma}\right) \\
& \leq\left\|W_{1}^{h}-\left(\tilde{W}_{1}^{h}+\theta\right)\right\|_{L^{\infty}\left(B_{R^{h}}\right)}+\left\|\tilde{W}_{1}^{h}\right\|_{L^{\infty}\left(B_{R^{h}}\right)}+\mathcal{O}\left(h^{\gamma}\right) \\
& \leq\left\|W_{1}^{h}-\left(\tilde{W}_{1}^{h}+\theta\right)\right\|_{L^{\infty}\left(\Omega^{h}\right)}+\left\|\tilde{W}_{1}^{h}\right\|_{L^{\infty}\left(B_{R^{h}}\right)}+\mathcal{O}\left(h^{\gamma}\right)_{h \rightarrow 0, h \in D}^{\underset{h}{\longrightarrow} 0}
\end{aligned}
$$


from which we get

$$
\lim _{\substack{h \rightarrow 0 \\ h \in D}}\left\|\tilde{V}^{h}-\theta\right\|_{L^{\infty}\left(B_{R^{h}}\right)}=0
$$

- Consider $1_{\Omega^{h}} \tilde{V}^{h}$ on $\mathbb{R}^{2} \backslash B_{\frac{R}{h}}$

$$
\begin{aligned}
\left\|1_{\Omega^{h}} \tilde{V}^{h}\right\|_{L^{\infty}\left(\mathbb{R}^{2} \backslash B_{\frac{R}{h}}\right)} & =\left\|\tilde{V}^{h}\right\|_{L^{\infty}\left(\Omega^{h} \backslash B_{\frac{R}{h}}\right)} \leq\left\|W_{1}^{h}\right\|_{L^{\infty}\left(\Omega^{h} \backslash B_{\frac{R}{h}}\right)}+\left\|W_{2}^{h}\right\|_{L^{\infty}\left(\Omega^{h} \backslash B_{\frac{R}{h}}\right)} \\
& \leq\left\|W_{1}^{h}\right\|_{L^{\infty}\left(\Omega^{h} \backslash B_{\frac{R}{h}}\right)}+\left\|W_{2}^{h}\right\|_{L^{\infty}\left(\Omega^{h}\right)}=\left\|W_{1}^{h}\right\|_{L^{\infty}\left(\Omega^{h} \backslash B_{\left.\frac{R}{h}\right)}\right.}+\mathcal{O}\left(h^{\gamma}\right) \\
& \leq\left\|W_{1}^{h}-\left(\tilde{W}_{1}^{h}+\theta\right)\right\|_{L^{\infty}\left(\Omega^{h} \backslash B_{\left.\frac{R}{h}\right)}\right.}+\left\|\tilde{W}_{1}^{h}+\theta\right\|_{L^{\infty}\left(\Omega^{h} \backslash B_{\frac{R}{h}}\right)}+\mathcal{O}\left(h^{\gamma}\right) \\
& \leq\left\|W_{1}^{h}-\left(\tilde{W}_{1}^{h}+\theta\right)\right\|_{L^{\infty}\left(\Omega^{h}\right)}+\left\|\tilde{W}_{1}^{h}+\theta\right\|_{L^{\infty}\left(\Omega^{h} \backslash B_{\frac{R}{h}}\right)}+\mathcal{O}\left(h^{\gamma}\right) \underset{h \rightarrow 0, h \in D}{\longrightarrow} 0
\end{aligned}
$$

which gives

$$
\lim _{\substack{h \rightarrow 0 \\ h \in D}}\left\|1_{\Omega^{h}} \tilde{V}^{h}\right\|_{L^{\infty}\left(\mathbb{R}^{2} \backslash B_{\frac{R}{h}}\right)}=0 .
$$

- Finally we consider $\tilde{V}^{h}$ on $B_{\frac{R}{h}} \backslash B_{R^{h}}$

$$
\begin{aligned}
\left\|\tilde{V}^{h}\right\|_{L^{\infty}\left(B_{\frac{R}{h}} \backslash B_{R^{h}}\right)} & \leq\left\|W_{1}^{h}-\left(\tilde{W}_{1}^{h}+\theta\right)\right\|_{L^{\infty}\left(\Omega^{h}\right)}+\left\|\tilde{W}_{1}^{h}+\theta\right\|_{L^{\infty}\left(B_{\frac{R}{h}} \backslash B_{R^{h}}\right)}+\left\|W_{2}^{h}\right\|_{L^{\infty}\left(\Omega^{h}\right)} \\
& \leq\left\|W_{1}^{h}-\left(\tilde{W}_{1}^{h}+\theta\right)\right\|_{L^{\infty}\left(\Omega^{h}\right)}+C+\left\|\tilde{W}_{1}^{h}\right\|_{L^{\infty}\left(B_{\frac{R}{h}} \backslash B_{R^{h}}\right)}+\left\|W_{2}^{h}\right\|_{L^{\infty}\left(\Omega^{h}\right)} .
\end{aligned}
$$

From the results of Lemma 4.1 and 4.2, we have

$$
\left\|\tilde{V}^{h}\right\|_{L^{\infty}\left(B_{\frac{R}{h}} \backslash B_{R^{h}}\right)} \leq C+\left\|\tilde{W}_{1}^{h}\right\|_{L^{\infty}\left(B_{\frac{R}{h}} \backslash B_{R^{h}}\right)}, \quad h \in D .
$$

Set $x \in B_{\frac{R}{h}} \backslash B_{R^{h}}$; proceeding as in Lemma 4.2, the inequality

$$
\begin{aligned}
\left|\tilde{W}_{1}^{h}(x)\right| & \leq \frac{\left(\ln \frac{1}{h}\right)^{-1}}{2 \pi} \sum_{\substack{i \leq N_{0} \\
\varepsilon_{i}^{h}<\varepsilon_{S}}} B_{i}^{h} \int_{B_{R^{h}}}|\ln | x-y \|\left|\tilde{\Psi}_{i}^{h}(y)\right|^{2} d y \\
& \leq C\left(\ln \frac{1}{h}\right)^{-1} \sum_{\substack{i \leq N_{0} \\
\varepsilon_{i}^{h}<\varepsilon_{S}}}\left(\left\|\ln || x-\left.y\left|\left\|_{L^{2}(B(x, 1))}\right\| \tilde{\Psi}_{i}^{h} \|_{L^{4}\left(\Omega^{h}\right)}^{2}+\ln \frac{2 R}{h} \int_{B_{R^{h} \backslash B(x, 1)}}\right| \tilde{\Psi}_{i}^{h}(y)\right|^{2} d y\right)\right. \\
& \leq C\left(\ln \frac{1}{h}\right)^{-1} \sum_{\substack{i \leq N_{0} \\
\varepsilon_{i}^{h}<\varepsilon_{S}}}\left(1+\ln \frac{2 R}{h}\left\|\tilde{\Psi}_{i}^{h}\right\|_{L^{2}\left(\Omega^{h}\right)}^{2}\right)
\end{aligned}
$$

holds, $C$ denoting at each step a positive constant. The leading asymptotic term at the r.h.s. of this expression is the product: $\left(\ln \frac{1}{h}\right)^{-1} \ln \frac{2 R}{h}$ which is a constant as $h \rightarrow 0$. The above considerations allow us to conclude that $\left.\tilde{V}^{h}\right|_{B_{\frac{R}{h}} \backslash B_{R^{h}}}$ is uniformly bounded for $h \in D$, i.e. it exists a positive constant $C$ such that

$$
\left\|\tilde{V}^{h}\right\|_{L^{\infty}\left(B_{\frac{R}{h}} \backslash B_{R^{h}}\right)} \leq C \quad \forall h \in D .
$$


As a direct consequence of (4.21), (4.22) and (4.23), $\left\{1_{\Omega^{h}} \tilde{V}^{h}\right\}_{h \in D}$ is bounded in the whole space uniformly w.r.t. $h$. Moreover, for any compact set $K \subset \mathbb{R}^{2}$, it exists $h_{K} \in D$ such that $K \subset B_{R^{h}}$ for all $h \in D \cap\left\{h \mid h<h_{K}\right\}$. This circumstance implies that

$$
\left\|\tilde{V}^{h}-\theta\right\|_{L^{\infty}(K)} \leq\left\|\tilde{V}^{h}-\theta\right\|_{L^{\infty}\left(B_{R^{h}}\right)} \underset{h \rightarrow 0, h \in D}{\longrightarrow} 0 .
$$

Corollary 4.4 Under the assumptions of Proposition 4.3, the family $\left(V^{h}\right)_{h \in D}$ converges strongly to 0 in $H_{0}^{1}(\Omega)$

$$
\left\|V^{h}\right\|_{H_{0}^{1}(\Omega)}=\mathcal{O}\left(\frac{1}{|\ln h|}\right) .
$$

Proof. The Poisson potentials at the classical and quantum scales are related by the change of variables: $\Omega \rightarrow \Omega^{h}$ and the relation (1.11). In $2 \mathrm{D}$, we have

$$
\left\|V^{h}\right\|_{H_{0}^{1}(\Omega)}=\left\|\tilde{V}^{h}\right\|_{H_{0}^{1}\left(\Omega^{h}\right)} .
$$

Projecting the Poisson equation for $\tilde{V}^{h}$ (the second one in (2.26)) over $\tilde{V}^{h}$ itself, we can estimate the $H_{0}^{1}$-norm of this function by

$$
\left\|\tilde{V}^{h}\right\|_{H_{0}^{1}\left(\Omega^{h}\right)}^{2}=|\ln h|^{-1} \sum_{\substack{i \leq N_{0} \\ \varepsilon_{i}^{h}<\varepsilon_{S}}} B_{i}^{h} \int_{\Omega^{h}}\left|\tilde{\Psi}_{i}^{h}\right|^{2} \tilde{V}^{h} d x \leq|\ln h|^{-1}\left\|1_{\Omega^{h}} \tilde{V}^{h}\right\|_{L^{\infty}\left(\mathbb{R}^{2}\right)} \sum_{\substack{i \leq N_{0} \\ \varepsilon_{i}^{h}<\varepsilon_{S}}} B_{i}^{h} .
$$

Following the result of Proposition 4.3, we have

$$
\left\|1_{\Omega^{h}} \tilde{V}^{h}\right\|_{L^{\infty}\left(\mathbb{R}^{2}\right)} \leq C \quad \forall h \in D,
$$

and, due to the boundedness of the coefficients $B_{i}^{h}$, it follows that

$$
\left\|V^{h}\right\|_{H_{0}^{1}(\Omega)}=\mathcal{O}\left(|\ln h|^{-1}\right) \text {. }
$$

As shown in Proposition 4.3, the limit potential at the quantum scale coincides with the constant value $\theta$ on compact sets. In the following Lemma we give a numerical estimate of this quantity.

Lemma 4.5 Let $e_{1}$ denotes the first eigenvalue of the Hamiltonian $H_{0}=-\Delta+U, D\left(H_{0}\right)=$ $H^{2}\left(\mathbb{R}^{d}\right)$. Under the assumptions of Proposition 4.3, the following conditions hold

$$
\begin{gathered}
\lim _{h \rightarrow 0} \inf \varepsilon_{i}^{h}>\varepsilon_{S} \quad \text { for } i>1, \\
\lim _{h \rightarrow 0} \varepsilon_{1}^{h}=e_{1}+\theta \quad \text { and } \quad \lim _{\substack{h \rightarrow 0 \\
h \in D}}|\ln h| f\left(\varepsilon_{1}^{h}\right)=2 \pi \theta .
\end{gathered}
$$

Proof. In what follows, $N_{1}$ denotes the integer

$$
N_{1}=\max \left\{i \mid \lim _{h \rightarrow 0} \inf \varepsilon_{i}^{h}=\varepsilon_{S}\right\},
$$

while $\left\{e_{i}\right\}_{i \leq N}$ is the point spectrum of $H_{0}$ and $H_{\theta}$ the operator

$$
H_{\theta}=-\Delta+U+\theta, \quad D\left(H_{\theta}\right)=H^{2}\left(\mathbb{R}^{2}\right)
$$

whose eigenvalues are

$$
e_{i}^{\theta}=e_{i}+\theta, \quad i=1, \ldots, N
$$


As in the proof of Proposition 3.3, it is possible to find a set $D \subset\left(0, h_{0}\right]$ such that condition (4.3) is verified and the following limit holds

$$
\lim _{\substack{h \rightarrow 0 \\ h \in D}} \varepsilon_{i \leq N_{1}}^{h}=\varepsilon_{S},
$$

with $N_{1} \geq 1$. Due to the above limit, one can set $h_{0}$ so small that $\varepsilon_{i \leq N_{1}}^{h}<\frac{\varepsilon_{S}}{2}, \forall h \in D$. This property allows us to apply the exponential estimate (A.17) for all $h \in \bar{D}$.

To obtain the results stated in (4.25) and (4.26), it is enough to prove that: $N_{1}=1$ and $\varepsilon_{S}=e_{1}+\theta$. The proof is articulated in three steps. We start showing that the eigenvalues $\varepsilon_{i \leq N_{1}}^{h}$ of the Hamiltonian $\tilde{H}^{h}$ asymptotically belong to the spectrum $\sigma\left(H_{\theta}\right)$ as $h \rightarrow 0$. Then, the limit

$$
\lim _{\substack{h \rightarrow 0 \\ h \in D}} \varepsilon_{i \leq N_{1}}^{h}=e_{1}^{\theta}
$$

is achieved. We conclude by using a spectral approximation argument.

- Consider the vector family: $u_{i \leq N_{1}}^{h}=\chi^{h} \tilde{\Psi}_{i \leq N_{1}}^{h}$, where $\chi^{h}(x)=\chi\left(h x+x_{0}\right)$ and $\chi \in C_{0}^{\infty}\left(\mathbb{R}^{2}\right)$ is such that: $\chi=1$ on $B_{\frac{R}{2}}$ and $\chi=0$ on $\mathbb{R}^{2} \backslash B_{R}$, for a radius $R>0$ verifying (4.7). Under these assumptions, the functions $u_{i \leq N_{1}}^{h}$ are in $H^{2}\left(\mathbb{R}^{2}\right)$ and we can consider the difference: $\left(H_{\theta}-\varepsilon_{i}^{h}\right) u_{i \leq N_{1}}^{h}$ which is expressed by

$$
\left(H_{\theta}-\varepsilon_{i}^{h}\right) u_{i}^{h}=-\left(\Delta \chi^{h}\right) \tilde{\Psi}_{i}^{h}-2 \nabla \chi^{h} \cdot \nabla \tilde{\Psi}_{i}^{h}-\chi^{h}\left(\tilde{V}^{h}-\theta\right) \tilde{\Psi}_{i}^{h} .
$$

The functions $\nabla \chi^{h}$ and $\Delta \chi^{h}$ are smooth and supported outside $B_{\frac{R}{2 h}}$. A direct application of estimate (A.17) gives

$$
\left\|\nabla \chi^{h} \cdot \nabla \tilde{\Psi}_{i}^{h}\right\|_{L^{2}\left(\mathbb{R}^{2}\right)}+\left\|\left(\Delta \chi^{h}\right) \tilde{\Psi}_{i}^{h}\right\|_{L^{2}\left(\mathbb{R}^{2}\right)} \leq C\|\chi\|_{C^{2}\left(\mathbb{R}^{2}\right)}\left(h+h^{2}\right) e^{-c_{0} \frac{R}{2 h}} \quad \forall h \in D .
$$

Next, we define $R^{h}=R \ln \frac{1}{h}$ and we suppose that $h_{0}$ is small enough so that (4.8) holds; using (A.17), equation (4.20) and the boundedness of $\left\{1_{\Omega^{h}} \tilde{V}^{h}\right\}_{h \in D}$ (obtained in Proposition 4.3) we have $\forall h \in D$

$$
\begin{aligned}
\left\|\chi^{h}\left(\tilde{V}^{h}-\theta\right) \tilde{\Psi}_{i}^{h}\right\|_{L^{2}\left(\mathbb{R}^{2}\right)}^{2} & \leq\|\chi\|_{L^{\infty}\left(\mathbb{R}^{2}\right)}^{2}\left(\int_{B_{R^{h}}}\left|\left(\tilde{V}^{h}-\theta\right) \tilde{\Psi}_{i}^{h}\right|^{2} d x+\int_{\Omega^{h} \backslash B_{R^{h}}}\left|\left(\tilde{V}^{h}-\theta\right) \tilde{\Psi}_{i}^{h}\right|^{2} d x\right) \\
& \leq C\left(\left\|\tilde{V}^{h}-\theta\right\|_{L^{\infty}\left(B_{R^{h}}\right)}^{2}+\int_{\Omega^{h}}\left|e^{c_{0}|x|} \tilde{\Psi}_{i}^{h}\right|^{2} d x e^{-2 c_{0} R^{h}}\right)
\end{aligned}
$$

and

$$
\lim _{\substack{h \rightarrow 0 \\ h \in D}}\left\|\chi^{h}\left(\tilde{V}^{h}-\theta\right) \tilde{\Psi}_{i}^{h}\right\|_{L^{2}\left(\mathbb{R}^{2}\right)}=0 .
$$

Combining equations (4.29) and the result above, it is possible to find a function $\alpha(h)$ independent of $i$ such that $\alpha(h) \rightarrow 0$ when $h \rightarrow 0$ and

$$
\left\|\left(H_{\theta}-\varepsilon_{i}^{h}\right) u_{i}^{h}\right\|_{L^{2}\left(\mathbb{R}^{2}\right)} \leq \alpha(h) \quad \forall h \in D .
$$

Let us denote with $d(\lambda, \sigma(H))$ the distance of $\lambda \in \mathbb{C}$ from the spectrum of an Hamiltonian $H$. Using a classical inequality for self-adjoint operators, we have

$$
\left\|u_{i}^{h}\right\|_{L^{2}\left(\mathbb{R}^{2}\right)} d\left(\varepsilon_{i}^{h}, \sigma\left(H_{\theta}\right)\right) \leq\left\|\left(H_{\theta}-\varepsilon_{i}^{h}\right) u_{i}^{h}\right\|_{L^{2}\left(\mathbb{R}^{2}\right)} .
$$

Due to the exponential decay relation (A.17) and the normalization condition (1.13), the mass of $\tilde{\Psi}_{i}^{h}$ concentrates around the origin. This circumstance allows us to obtain a uniform lower bound for $\int_{B_{\frac{R}{2 h}}}\left|\tilde{\Psi}_{i}^{h}\right|^{2} d x$ (see e.g. (2.27) in Lemma 2.7), and

$$
\left\|u_{i}^{h}\right\|_{L^{2}\left(\mathbb{R}^{2}\right)} \geq \frac{1}{2}
$$


for all $h \in D$ and $h_{0}$ small enough. Previous relations lead us to the following result

$$
d\left(\varepsilon_{i}^{h}, \sigma\left(H_{\theta}\right)\right) \leq 2\left\|\left(H_{\theta}-\varepsilon_{i}^{h}\right) u_{i}^{h}\right\|_{L^{2}\left(\mathbb{R}^{2}\right)} \underset{h \rightarrow 0, h \in D}{\longrightarrow} 0 .
$$

- The above condition and the limit (4.28), imply that $\varepsilon_{S} \in \sigma\left(H_{\theta}\right)$ and, in particular

$$
\varepsilon_{S}=e_{k}^{\theta}
$$

for some $k \geq 1$. On the other hand, following the same line as in the previous step, it is possible to show that

$$
\lim _{\substack{h \rightarrow 0 \\ h \in D}} d\left(e_{1}^{\theta}, \sigma\left(\tilde{H}_{h}\right)\right)=0 .
$$

Here it follows a brief sketch of the proof. Let $\phi_{1} \in H^{2}\left(\mathbb{R}^{2}\right)$ denote the first normalized eigenvector of $H_{\theta}$. Exploiting the Agmon distance on $\mathbb{R}^{2}$ associated with the potential $\left(U-\frac{e_{1}}{2}\right)$ from a point $x$ to the well $\omega$, it is possible to recover the quantum scale decay estimate

$$
\int_{\mathbb{R}^{2}}\left|e^{c_{0}|x|} \phi_{1}\right|^{2} d x+\int_{\mathbb{R}^{2}}\left|e^{c_{0}|x|} \nabla \phi_{1}\right|^{2} d x \leq C
$$

for suitable positive constants $c_{0}$ and $C$ (this is the analogous of relation (A.6) in the whole space case, we refer to Proposition 6.4 in [6] for this point). Setting $v^{h}=\chi^{h} \phi_{1}$, we have $v^{h} \in D\left(\tilde{H}^{h}\right)$ and

$$
\left(\tilde{H}^{h}-e_{1}^{\theta}\right) v^{h}=-\left(\Delta \chi^{h}\right) \phi_{1}-2 \nabla \chi^{h} \cdot \nabla \phi_{1}+\chi^{h}\left(\tilde{V}^{h}-\theta\right) \phi_{1} .
$$

The estimate (4.34) allows to apply the same scheme as in the previous case. Therefore we have

$$
\left\|v^{h}\right\|_{L^{2}\left(\mathbb{R}^{2}\right)} \geq \frac{1}{2} \quad \text { and } \quad \lim _{\substack{h \rightarrow 0 \\ h \in D}}\left\|\left(\tilde{H}^{h}-e_{1}^{\theta}\right) v^{h}\right\|_{L^{2}\left(\mathbb{R}^{2}\right)}=0
$$

from which the relation (4.33) directly follows.

Using the constraints (4.28) and (4.32), a direct consequence of (4.33) is

$$
\lim _{\substack{h \rightarrow 0 \\ h \in D}} \varepsilon_{i \leq N_{1}}^{h}=\varepsilon_{S}=e_{1}+\theta .
$$

- Let $b=\frac{e_{2}^{\theta}-e_{1}^{\theta}}{2}>0$. The unique spectral point of $H_{\theta}$ in the interval $I:=\left[e_{1}^{\theta}-b, e_{1}^{\theta}+b\right]$ is $e_{1}^{\theta}$; moreover, $H_{\theta}$ has no spectrum in $\{x, 0<d(x, I)<b\}$

$$
\sigma\left(H_{\theta}\right) \cap((I+B(0, b)) \backslash I)=\varnothing .
$$

From the previous point, we know that $\varepsilon_{i \leq N_{1}}^{h}$ definitely belong to $I$ when $h \in D$. Next we consider the vector space $E$ spanned by the family $\left(u_{i}^{h}\right)_{i \leq N_{1}}$ and the spectral subspace $F$ associated with $\sigma\left(H_{\theta}\right) \cap I$. By construction, $F$ coincides with the first eigenspace of the operator $H_{\theta}$. Using (A.17), it can be easily shown that the matrix $M=\left(\left(u_{i}^{h}, u_{j}^{h}\right)_{L^{2}\left(\mathbb{R}^{2}\right)}\right)_{1 \leq i, j \leq N_{1}}$ verifies

$$
M=I+o(1)
$$

when $h \rightarrow 0, h \in D$. Then, from Theorem 2.4 in [9] and the relation (4.30), the distance $d(E, F)$ (definition given in [9]) is estimated by

$$
d(E, F) \leq\left(\frac{N_{1}}{\lambda_{\min }}\right)^{\frac{1}{2}} \frac{2 \alpha(h)}{b} \quad \forall h \in D,
$$

where $\lambda_{\text {min }}$ denotes the smallest eigenvalue of $M$. Equation (4.38) implies

$$
\lambda_{\min }=1+o(1) \geq \frac{1}{2} \quad \forall h \in D .
$$


Using the above equation and the limit condition $\lim _{h \rightarrow 0} \alpha(h)=0$ in (4.39), we get

$$
d(E, F)<1
$$

for all $h \in D$ with $h_{0}$ small enough. This last condition allows us to state that the map $\left.\Pi_{F}\right|_{E}$ : $E \rightarrow F$ is injective (e.g. in Lemma 1.3 in [9]), from which the condition $N_{1}=1$, and then (4.25) follow. For a set $D$ such that condition (4.3) holds, the last $\operatorname{limit}_{\substack{h \in D \\ \lim _{h \rightarrow 0}}}|\ln h| f\left(\varepsilon_{1}^{h}\right)=2 \pi \theta$ is a simple rewriting of the definition (4.16) of $\theta$ when (4.25) is true.

We conclude this Section giving the proof of Theorem 1.3

Proof of Theorem 1.3. Let $D, 0 \in \bar{D}$, be any set for which the condition (4.3) is verified. The constant $\theta$ related to $D$ is defined by

$$
\theta=\frac{1}{2 \pi} \sum_{i \leq N_{0}} B_{i}, \quad \lim _{\substack{h \rightarrow 0 \\ h \in D}} B_{i}^{h}=B_{i}
$$

Due to the relations (2.20) and (4.26), this constant is uniquely determined from the data of the problem

$$
\theta=\varepsilon_{S}-e_{1}
$$

Assume now that the second statement is not true. Then there exists a constant $\kappa>0$, a set $S \subset\left(0, h_{0}\right], 0 \in \bar{S}$ and a strictly positive constant $c$ such that $\left\|\tilde{V}^{h}-\theta\right\|_{L^{\infty}(|x| \leq-\kappa \ln h)} \geq c$ for all $h \in S$. On the other hand, the boundedness of the coefficients stated in Lemma 2.7 allows us to extract a subset $D \subset S, 0 \in \bar{D}$, such that the condition (4.3) is verified. Then the Proposition 4.3 brings a contradiction. We have proved ad absurdum, the second statement of Theorem 1.3.

The third statement is also proved by using an ad absurdum argument. Out of any infinite set $S \subset\left(0, h_{0}\right]$ with $0 \in \bar{S}$ which contradicts the statement, a subset $D \subset S$ with $0 \in \bar{D}$ can be extracted so that (4.3) holds. Then the relation (4.5) implies the $L_{2}$ estimate. Moreover Lemma 4.5 says that $\varepsilon_{i}^{h}>\varepsilon_{S}$ for $i \geq 2$ and $h$ small enough and provides the asymptotic behaviour of $\varepsilon_{1}^{h}$ and $f\left(\varepsilon_{1}^{h}\right)$ as $h \rightarrow 0, h \in D$. Thus the subset $S$ cannot exist.

The first statement is a direct consequence of the second one combined with Corollary 4.4.

Acknowledgements The authors wish to acknowledge the contribution of Naoufel Ben Abdallah and Florian Méhats, whose advice and remarks aided in the completion of this study.

\section{A Agmon estimates}

Agmon estimates form a standard technical tool in evaluating the rate of decay of eigenfunctions far from the interaction support. In what follows we apply this technique to the case of the Schrödinger Poisson system with a squeezing quantum well; in particular we give some useful decay estimates for those stationary states - on both the classical and the quantum scales - related to the energies below some negative energy. Let us first recall the definition of the Agmon distance associated with a smooth potential $V$, defined on $D \subset \mathbb{R}^{d}$, and to the energy $E$

$$
d_{V-E}(x, y)=\inf _{\gamma \in \mathcal{P}_{x, y}} \int_{0}^{1} \sqrt{(V(\gamma(s))-E)_{+}}\left|\gamma^{\prime}(s)\right| d s
$$

where $\mathcal{P}_{x, y}$ denotes the set of all regular curves connecting $x$ and $y$ in $D$. We have the classical property

$$
\left|\nabla_{x} d_{V-E}(x, y)\right|^{2} \leq(V-E)_{+}(x) \text { a.e. }
$$

which is still true if we consider the Agmon distance from a set of points $\omega$ (e.g. in [8])

$$
d_{V-E}(x, \omega)=\inf _{y \in \omega} d_{V-E}(x, y) .
$$


Lemma A.1 (Agmon estimates) Let $\varepsilon_{i}^{h}$ be a spectral point of the (unitarily equivalent) Hamiltonians

$$
H^{h}=-h^{2} \Delta+U^{h}+V^{h}
$$

and

$$
\tilde{H}^{h}=-\Delta+U+\tilde{V}^{h}
$$

placed below some negative energy: $\varepsilon_{i}^{h}<\varepsilon$ where $\varepsilon \in\left(-\|U\|_{L^{\infty}}, 0\right)$. The related stationary states - $\Psi_{i}^{h}$ and $\tilde{\Psi}_{i}^{h}$ - admit the estimates

$$
\begin{gathered}
\left\|h \nabla\left(e^{\phi / h} \Psi_{i}^{h}\right)\right\|_{L^{2}(\Omega)}+\left\|e^{\phi / h} \Psi_{i}^{h}\right\|_{L^{2}(\Omega)} \leq C, \\
\left\|e^{\tilde{\phi}} \tilde{\Psi}_{i}^{h}\right\|_{H^{1}\left(\Omega^{h}\right)} \leq \tilde{C},
\end{gathered}
$$

where $C$ and $\tilde{C}$ are suitable positive constants, $\phi$ and $\tilde{\phi}$ are the Agmon distances

$$
\begin{gathered}
\phi(x)=(1-\delta) d_{U^{h}-\varepsilon}\left(x, \omega^{h}\right), \quad x \in \Omega \\
\tilde{\phi}(x)=(1-\delta) d_{U-\varepsilon}(x, \omega), \quad x \in \Omega^{h}
\end{gathered}
$$

$\delta$ is a positive parameter smaller than 1 , while $\omega^{h}$ and $\omega$ are the support of $U^{h}$ and $U$ respectively.

Proof. We start considering the problem at the quantum scale. We use the relation (see for instance Theorem 1.1 in [9])

$$
\int_{\Omega^{h}}\left|\nabla\left(e^{\varphi} u\right)\right|^{2} d x+\int_{\Omega^{h}}\left(V-|\nabla \varphi|^{2}\right) e^{2 \varphi} u^{2} d x=\int_{\Omega^{h}} e^{2 \varphi}(-\Delta+V) u \cdot u d x .
$$

Setting $u=\tilde{\Psi}_{i}^{h}, \varphi=\tilde{\phi}$ and $V=U+\tilde{V}^{h}-\varepsilon_{i}^{h}$, we get

$$
\int_{\Omega^{h}}\left|\nabla\left(e^{\tilde{\phi}} \tilde{\Psi}_{i}^{h}\right)\right|^{2} d x=-\int_{\Omega^{h}}\left(U+\tilde{V}^{h}-\varepsilon_{i}^{h}-|\nabla \tilde{\phi}|^{2}\right) e^{2 \phi}\left(\tilde{\Psi}_{i}^{h}\right)^{2} d x
$$

and, due to $(2.2)$,

$$
\int_{\Omega^{h}}\left|\nabla\left(e^{\tilde{\phi}} \tilde{\Psi}_{i}^{h}\right)\right|^{2} d x \leq-\int_{\Omega^{h}}\left(U-\varepsilon_{i}^{h}-|\nabla \tilde{\phi}|^{2}\right) e^{2 \tilde{\phi}}\left(\tilde{\Psi}_{i}^{h}\right)^{2} d x .
$$

Next we follow the same line as in Proposition 3.3.1 of [8] and introduce the set

$$
\Omega_{h, \delta}^{+}=\left\{x \in \Omega^{h} \mid U-\varepsilon \geq \delta\right\},
$$

where $\delta$ is a small positive parameter. Relation (A.11) can be written as

$\int_{\Omega^{h}}\left|\nabla\left(e^{\tilde{\phi}} \tilde{\Psi}_{i}^{h}\right)\right|^{2} d x+\int_{\Omega_{h, \delta}^{+}}\left(U-\varepsilon_{i}^{h}-|\nabla \tilde{\phi}|^{2}\right) e^{2 \tilde{\phi}}\left(\tilde{\Psi}_{i}^{h}\right)^{2} d x \leq-\int_{\Omega_{h, \delta}^{-}}\left(U-\varepsilon_{i}^{h}-|\nabla \tilde{\phi}|^{2}\right) e^{2 \tilde{\phi}}\left(\tilde{\Psi}_{i}^{h}\right)^{2} d x$

with $\Omega_{h, \delta}^{-}=\Omega^{h} \backslash \Omega_{h, \delta}^{+}$. The function $U-\varepsilon_{i}^{h}-|\nabla \tilde{\phi}|^{2}$ admits the following estimates

- The domain $\Omega_{h, \delta}^{-}$is inside the support of $U$; therefore, in this region, we have $\tilde{\phi}=0$, $U-\varepsilon_{i}^{h}-|\nabla \tilde{\phi}|^{2}=U-\varepsilon_{i}^{h}$ and

$$
\sup _{x \in \Omega_{h, \delta}^{-}}-\left(U-\varepsilon_{i}^{h}-|\nabla \tilde{\phi}|^{2}\right) \leq\|U\|_{L^{\infty}} .
$$


- In $\Omega_{h, \delta}^{+}$; applying (A.2), we have

$$
\begin{gathered}
U-\varepsilon_{i}^{h}-|\nabla \tilde{\phi}|^{2} \geq U-\varepsilon_{i}^{h}-(1-\delta)^{2}(U-\varepsilon) \\
\geq(U-\varepsilon)\left(2 \delta-\delta^{2}\right) \geq 2 \delta^{2}-\delta^{3} .
\end{gathered}
$$

For $\delta<\min \{1,|\varepsilon|\}$, it follows

$$
\inf _{x \in \Omega_{h, \delta}^{+}}\left(U-\varepsilon_{i}^{h}-|\nabla \tilde{\phi}|^{2}\right)>\delta^{2} .
$$

Replacing (A.13) and (A.14) into (A.12) and taking into account the condition $\left\|\tilde{\Psi}_{i}^{h}\right\|_{L^{2}\left(\Omega^{h}\right)}=1$, we obtain

$$
\int_{\Omega^{h}}\left|\nabla\left(e^{\tilde{\phi}} \tilde{\Psi}_{i}^{h}\right)\right|^{2} d x+\delta^{2} \int_{\Omega_{h, \delta}^{+}} e^{2 \tilde{\phi}}\left(\tilde{\Psi}_{i}^{h}\right)^{2} d x \leq\|U\|_{L^{\infty}}
$$

and

$$
\int_{\Omega^{h}}\left|\nabla\left(e^{\tilde{\phi}} \tilde{\Psi}_{i}^{h}\right)\right|^{2} d x+\delta^{2} \int_{\Omega^{h}} e^{2 \tilde{\phi}}\left(\tilde{\Psi}_{i}^{h}\right)^{2} d x \leq\|U\|_{L^{\infty}}+\delta^{2} \int_{\Omega_{h, \delta}^{-}} e^{2 \tilde{\phi}}\left(\tilde{\Psi}_{i}^{h}\right)^{2} d x \leq\|U\|_{L^{\infty}}+\delta^{2} .
$$

This gives the estimate (A.6).

The analogous result at the classical scale (A.5) can be easily achieved making use of the change of variables: $\Omega^{h} \rightarrow \Omega$, and taking into account the relations (1.8) and

$$
\frac{1}{h} d_{U^{h}-\varepsilon}\left(x, \omega^{h}\right)=d_{U-\varepsilon}\left(\frac{x-x_{0}}{h}, \omega\right), \quad x \in \Omega .
$$

Remark A.2 The previous Lemma allows us to state that the stationary states related to negative energies show an exponential decay outside the support of the potential well, which is given by $\omega^{h}$ or $\omega$ depending on the description adopted. In particular, for $\varepsilon<0$, we notice that the corresponding Agmon metric

$$
\left(U^{h}-\varepsilon\right)_{+}^{\frac{1}{2}} d x \quad \text { and } \quad(U-\varepsilon)_{+}^{\frac{1}{2}} d x
$$

identifies with the Euclidian one

$$
|\varepsilon|^{\frac{1}{2}} d x
$$

in the open domains $x \in \mathbb{R}^{d} \backslash \bar{\omega}^{h}$ and $x \in \mathbb{R}^{d} \backslash \bar{\omega}$ respectively. It follows that, in these regions, $\phi(x)$ and $\tilde{\phi}(x)$ are strictly positive functions increasing, for $|x| \rightarrow \infty$, as the standard Euclidean distance in $\mathbb{R}^{d}$. This property can be rephrased by the following Lemma.

Lemma A.3 The functions $\phi$ and $\tilde{\phi}$, defined in (A.7)-(A.8), admit the following estimate

$$
\begin{gathered}
c_{0}\left|x-x_{0}\right|-h c_{1} \leq \phi(x) \leq c_{2}\left|x-x_{0}\right|, \quad x \in \Omega \\
c_{0}|x|-c_{1} \leq \tilde{\phi}(x) \leq c_{2}|x|, \quad x \in \Omega^{h}
\end{gathered}
$$

where $c_{i}, i=0,1,2$, are suitable positive constants.

Corollary A.4 Set $\varepsilon \in\left(-\|U\|_{L^{\infty}}, 0\right)$. For $\varepsilon_{i}^{h}<\varepsilon$, the following estimates hold

$$
\begin{gathered}
\left\|e^{c_{0}|\cdot|} \tilde{\Psi}_{i}^{h}\right\|_{L^{2}\left(\Omega^{h}\right)}+\left\|e^{c_{0}|\cdot|} \nabla \tilde{\Psi}_{i}^{h}\right\|_{L^{2}\left(\Omega^{h}\right)} \leq C, \\
\left\|e^{c_{0}|\cdot|} \tilde{\Psi}_{i}^{h}\right\|_{L^{p}\left(\Omega^{h}\right)} \leq C^{\prime},
\end{gathered}
$$

with: $p \in[1,6]$ in dimension $d=3$ or $p \in[1,+\infty)$ in dimension $d=2$, and for suitable positive constants $C, C^{\prime}$. 
Proof. As a direct consequence of the estimate (A.6) and the inequality (A.16), it follows that the $L^{2}$-norm of the function $e^{c_{0}|\cdot|} \tilde{\Psi}_{i}^{h}$ is uniformly bounded w.r.t. $h$.

For what concern the second contribution in (A.17), we notice that

$$
e^{c_{0}|\cdot|-c_{1}}\left|\nabla \tilde{\Psi}_{i}^{h}\right| \leq\left|e^{\tilde{\phi}} \nabla \tilde{\Psi}_{i}^{h}\right|=\left|\nabla\left(e^{\tilde{\phi}} \tilde{\Psi}_{i}^{h}\right)-\left(\nabla e^{\tilde{\phi}}\right) \tilde{\Psi}_{i}^{h}\right| .
$$

The term $\nabla e^{\tilde{\phi}}$ at the r.h.s. is pointwise bounded by

$$
\left|\nabla e^{\tilde{\phi}}\right| \leq(U-\varepsilon)_{+}^{\frac{1}{2}} e^{\tilde{\phi}}
$$

as it comes from (A.2). Then, using once more the relation (A.6), we obtain

$$
e^{-c_{1}}\left\|e^{c_{0}|\cdot|} \nabla \tilde{\Psi}_{i}^{h}\right\|_{L^{2}\left(\Omega^{h}\right)} \leq\left\|\nabla\left(e^{\tilde{\phi}} \tilde{\Psi}_{i}^{h}\right)\right\|_{L^{2}\left(\Omega^{h}\right)}+\sup _{x \in \Omega^{h}}(U-\varepsilon)_{+}^{\frac{1}{2}}\left\|e^{\tilde{\phi}} \tilde{\Psi}_{i}^{h}\right\|_{L^{2}\left(\Omega^{h}\right)} \leq C .
$$

From the continuous injection $H^{1} \hookrightarrow L^{p}$ and the Agmon estimate (A.6), we get

$$
\left\|e^{\tilde{\phi}} \tilde{\Psi}_{i}^{h}\right\|_{L^{p}\left(\Omega^{h}\right)} \leq C^{\prime}
$$

Then, relation (A.18) easily follows taking into account the inequality $e^{p\left(c_{0}|\cdot|-c_{1}\right)}\left|\tilde{\Psi}_{i}^{h}\right|^{p} \leq\left|e^{\tilde{\phi}} \tilde{\Psi}_{i}^{h}\right|^{p}$.

\section{B Further technical tools}

Here is a basic result of functional analysis which is used in Section 3.2.

Lemma B.1 Let $H_{0}$ be a closed self-adjoint operator on a Hilbert space $\mathcal{H}$ with $\inf \sigma\left(H_{0}\right)=\varepsilon_{0}>$ $-\infty$ and $\inf \sigma_{e s s}\left(H_{0}\right)=\varepsilon_{0, e s s}$. Assume that $E$ is a real Banach space of symmetric relatively bounded perturbations with relative bound 0 of $H_{0}$ with the estimate

$$
\forall \delta>0, \exists C_{\delta}>0 ; \forall W \in E, \forall \psi \in D\left(H_{0}\right), \quad\|W \psi\| \leq \delta\|W\|_{E}\left\|H_{0} \psi\right\|+C_{\delta}\|W\|_{E}\|\psi\| .
$$

Then for any $W \in E$, the operator $H_{0}+W$ is self-adjoint with domain $D\left(H_{0}+W\right)=D\left(H_{0}\right)$, bounded from below and the mapping $\varepsilon: E \ni W \rightarrow \varepsilon(W)=\inf \sigma\left(H_{0}+W\right) \in \mathbb{R}$ is continuous. Moreover if any $W \in E$ is a relatively compact perturbation of $H_{0}$, then $\sigma_{\text {ess }}\left(H_{0}+W\right)=\sigma_{\text {ess }}\left(H_{0}\right)$ for any $W \in E$ and the mapping $\varepsilon$ is real analytic on the open set $\left\{W \in E, \varepsilon(W)<\varepsilon_{0, e s s}\right\}$.

Proof. Any $W \in E$ is a relatively bounded perturbation with bound less than 1 of $H_{0}$. Hence $H_{0}+W$ is self-adjoint with $D\left(H_{0}+W\right)=D\left(H_{0}\right)$. The mapping $\varepsilon$ is well defined from $E$ to $\mathbb{R} \cup\{-\infty\}$ by

$$
\varepsilon(W)=\inf _{\varphi \in \mathcal{H} \backslash\{0\}} \frac{\left(\varphi,\left(H_{0}+W\right) \varphi\right)}{\|\varphi\|^{2}}
$$

is concave as an infimum of affine functions. It is continuous when it is locally bounded. This is again a consequence of our accurate relative boundedness with the second resolvent formula. Write for $\lambda \in \mathbb{R}$ and $\lambda \geq 2\left|\varepsilon_{0}\right|+1$

$$
\left(H_{0}+W+\lambda\right)^{-1}=\left[\operatorname{Id}+\left(H_{0}+\lambda\right)^{-1} W\right]^{-1}\left(H_{0}+\lambda\right)^{-1}
$$

with

$$
\left\|\left(H_{0}+\lambda\right)^{-1} W\right\|=\left\|W\left(H_{0}+\lambda\right)^{-1}\right\| \leq 2 \delta\|W\|_{E}+\frac{C_{\delta}}{\lambda-\left|\varepsilon_{0}\right|}\|W\|_{E} .
$$

For $\|W\|_{E} \leq R$, and by taking $\lambda>2\left|\varepsilon_{0}\right|+1+3 R C_{1 /(6 R)}$ and $\delta=1 /(6 R)$, the resolvent $\left(\lambda+H_{0}+\right.$ $W)^{-1}$ exists and equals the series

$$
\left(H_{0}+W+\lambda\right)^{-1}=\sum_{k=0}^{\infty}(-1)^{k}\left[\left(\lambda+H_{0}\right)^{-1} W\right]^{k}\left(\lambda+H_{0}\right)^{-1} \quad \text { in } \mathcal{L}(\mathcal{H}) .
$$


Hence $\varepsilon(W) \geq-1-2\left|\varepsilon_{0}\right|-3 R C_{1 /(6 R)}$ when $\|W\|_{E} \leq R$. This ends the proof of the continuity. The additional relative compactness assumption with the Weyl and the Kato-Rellich theorems (see [17]-IV) yield the second statement.

\section{References}

[1] A. Ambrosetti, G. Prodi, A Premier of Nonlinear Analysis, Cambridge University Press, Cambridge, 1993.

[2] V. Bonnaillie, F. Nier, Y. Patel. Far from equlibrium steady states of 1D-Schrödinger-Poisson systems with quantum wells I. Prépublication IRMAR, 07-02 (2007), to appear in Ann. I.H.P. An. Non Linéaire.

[3] V. Bonaillie, F. Nier, Y. Patel. Far from equlibrium steady states of 1D-Schrödinger-Poisson systems with quantum wells II. Prépublication IRMAR, 07-03 (2007), to appear in J. Math. Soc. of Japan.

[4] V. Bonaillie, F. Nier, Y. Patel. Computing the steady states for an asymptotic model of quantum transport in resonant heterostructures. Journal of Computational Physics, 219(2), 644-670, 2006.

[5] H. Brézis, Analyse fonctionnelle, Théories et applications, Masson, 1983.

[6] M. Dimassi, J. Sjötrand, Spectral Asymptotics in the semi-classical limit, Cambridge University Press, 1999.

[7] I. Ekeland, R. Temam, Convex analysis and variational problems. Classics in Applied Mathematics, 28. Society for Industrial and Applied Mathematics, Philadelphia, 1999.

[8] B. Helffer, Semi-Classical Analysis for the Schrödinger Operator and Applications, Lecture Notes in Mathematics, Springer-Verlag, vol.13.

[9] B. Helffer, J. Sjötrand, Multiple wells in the semi-classical limit I, Comm. In PDE, 9(4), 337-348, 1984.

[10] G. Jona-Lasinio, C. Presilla, J. Sjöstrand. On Schrödinger equations with concentrated nonlinearities. Ann. Phys., 240(1), 1-21, 1995.

[11] J. Kastrup et al. Self-oscillations of domains in doped GaAs-Al-As superlatices. Phys. Rev. B, 52(19), 13761-13764, 1995.

[12] F. Nier, A variational formulation of Schrödinger-Poisson systems in dimension $d \leq 3$, Comm. Part. Diff. Eq. 18(7-8), 1125-1147, 1993.

[13] F. Nier, Accurate WKB approximation for a 1D problem with low regularity, Serdica Mathematical Journal, 34(1) 113-126, 2008.

[14] L. Nirenberg, On elliptic partial differential equations, Ann. Scuola Norm. Sup. Pisa, 13, 116-162, 1959.

[15] J. Pöschel, E. Trubowitz, Inverse Spectral Theory, Academic Press, Boston, 1987.

[16] C. Presilla, J. Sjöstrand. Transport properties in resonant tunneling heterostructures. J. Math. Phys., 37(10), 4816-4844, 1996.36.

[17] M. Reed, B. Simon, Methods of Modern Mathematical Physics Vol. IV: Analysis of Operators, Academic Press, New York, 1978.

[18] B. Simon, Trace Ideals and their Applications, Cambridge University Press, London, 1979. 